TRANSACTIONS OF THE

AMERICAN MATHEMATICAL SOCIETY

Volume 362, Number 3, March 2010, Pages 1599-1617

S 0002-9947(09)04802-8

Article electronically published on October 6, 2009

\title{
A NEW APPROACH TO CLASSIFICATION OF INTEGRAL QUADRATIC FORMS OVER DYADIC LOCAL FIELDS
}

\author{
CONSTANTIN N. BELI
}

\begin{abstract}
In 1963, O'Meara solved the classification problem for lattices over dyadic local fields in terms of Jordan decompositions. In this paper we translate his result in terms of good BONGs. BONGs (bases of norm generators) were introduced in 2003 as a new way of describing lattices over dyadic local fields. This result and the notions we introduce here are a first step towards a solution of the more difficult problem of representations of lattices over dyadic fields.
\end{abstract}

\section{INTRODUCTION}

Since the main result of this paper is given in terms of BONGs, which were introduced in [1, we now give a reminder of some of the definitions and results in that paper which we will use here.

Throughout this paper $F$ is a dyadic local field, $\mathcal{O}$ the ring of integers, $\mathfrak{p}$ the prime ideal, $\mathcal{O}^{\times}:=\mathcal{O} \backslash \mathfrak{p}$ the group of units, $e:=\operatorname{ord} 2$ and $\pi$ is a fixed prime element. For $a \in \dot{F}$ we denote its quadratic defect by $\mathfrak{d}(a)$ and let $\Delta=1-4 \rho$ be a fixed unit with $\mathfrak{d}(\Delta)=4 \mathcal{O}$.

We denote by $d: \dot{F} / \dot{F}^{2} \longrightarrow \mathbb{N} \cup\{\infty\}$ the order of the "relative quadratic defect" $d(a)=\operatorname{ord} a^{-1} \mathfrak{d}(a)$. If $a=\pi^{R} \varepsilon$, with $\varepsilon \in \mathcal{O}^{\times}$, then $d(a)=0$ if $R$ is odd and $d(a)=d(\varepsilon)=\operatorname{ord} \mathfrak{d}(\varepsilon)$ if $R$ is even. Thus $d(\dot{F})=\{0,1,3, \ldots, 2 e-1,2 e, \infty\}$. This function satisfies the domination principle $d(a b) \geq \min \{d(a), d(b)\}$.

If $\alpha$ is a positive integer then $\left(1+\mathfrak{p}^{\alpha}\right) \dot{F}^{2}=\{a \in \dot{F} \mid d(a) \geq \alpha\}$ and $\left(1+\mathfrak{p}^{\alpha}\right) \mathcal{O}^{\times 2}=$ $\left\{a \in \mathcal{O}^{\times} \mid d(a) \geq \alpha\right\}$. For convenience we set $\left(1+\mathfrak{p}^{\alpha}\right) \dot{F}^{2}:=\{a \in \dot{F} \mid d(a) \geq \alpha\}$ and $\left(1+\mathfrak{p}^{\alpha}\right) \mathcal{O}^{\times 2}:=\left\{a \in \mathcal{O}^{\times} \mid d(a) \geq \alpha\right\}$ for any $\alpha \in \mathbb{R} \cup\{\infty\}$. Thus $\left(1+\mathfrak{p}^{\alpha}\right) \dot{F}^{2}=\dot{F}^{2}$ for $\alpha>2 e$ and $\left(1+\mathfrak{p}^{\alpha}\right) \dot{F}^{2}=\dot{F}$ for $\alpha \leq 0$. If $d$ is the smallest element in $d(\dot{F})$ s.t. $\alpha \leq d$ then $\left(1+\mathfrak{p}^{\alpha}\right) \dot{F}^{2}=\left(1+\mathfrak{p}^{d}\right) \dot{F}^{2}$.

We denote by $(\cdot, \cdot)_{\mathfrak{p}}: \dot{F} / \dot{F}^{2} \times \dot{F} / \dot{F}^{2} \longrightarrow\{ \pm 1\}$ the Hilbert symbol, which is a non-degenerate bilinear symmetric form.

If $a \in \dot{F}$, we denote by $\mathrm{N}(a)$ the norm group $\mathrm{N}(F(\sqrt{a}) / F)=\left\{b \in \dot{F} \mid(a, b)_{\mathfrak{p}}=\right.$ $1\}$. If $b \in \dot{F}$ and $d(a)+d(b)>2 e$ then $(a, b)_{\mathfrak{p}}=1$. However if $\alpha \notin \dot{F}^{2}$ then there is $b \in \dot{F}$ with $d(b)=2 e-d(a)$ s.t. $(a, b)_{\mathfrak{p}}=-1$. (For $d(a)$ odd this is just 3 , Lemma 3]. If $d(a)=2 e$ and $b \in \dot{F}$ is arbitrary with $d(b)=0$ then $a \in \Delta \dot{F}^{2}$ and ord $b$ is odd

Received by the editors November 14, 2006 and, in revised form, April 8, 2008.

2000 Mathematics Subject Classification. Primary 11E08.

This research was partially supported by the Contract 2-CEx06-11-20.

In Beli (2006) this paper was announced under the title "BONG version of O'Meara's 93:28 theorem". We changed the title at the referee's suggestion.

(C)2009 American Mathematical Society Reverts to public domain 28 years from publication 
so $(a, b)_{\mathfrak{p}}=-1$. Similarly if $d(a)=0$ and $d(b)=2 e$ we have $(a, b)_{\mathfrak{p}}=-1$.) Thus $\left(1+\mathfrak{p}^{\alpha}\right) \dot{F}^{2} \subseteq \mathrm{N}(a)$ iff $\alpha+d(a)>2 e$.

An element $x$ of a lattice $L$ is called a norm generator of $L$ if $\mathfrak{n} L=Q(x) \mathcal{O}$. A sequence $x_{1}, \ldots, x_{n}$ of vectors in $F L$ is called a basis of norm generators (BONG) for $L$ if $x_{1}$ is a norm generator for $L$ and $x_{2}, \ldots, x_{n}$ is a BONG for $p r_{x_{1}^{\perp}} L$. A BONG uniquely determines a lattice, so if $x_{1}, \ldots, x_{n}$ is a BONG for $L$, we will write $L=\prec x_{1}, \ldots, x_{n} \succ$. If moreover $Q\left(x_{i}\right)=a_{i}$ we say that $L \cong \prec a_{1}, \ldots, a_{n} \succ$ relative to the BONG $x_{1}, \ldots, x_{n}$. If $L \cong \prec a_{1}, \ldots, a_{n} \succ$ then $\operatorname{det} L=a_{1} \cdots a_{n}$.

If $x_{1}, \ldots, x_{n}$ are mutually orthogonal vectors with $Q\left(x_{i}\right)=a_{i}, L=\mathcal{O} x_{1} \perp \cdots \perp$ $\mathcal{O} x_{n}$ and $V=F x_{1} \perp \cdots \perp F x_{n}$ then we say that $L \cong\left\langle a_{1}, \ldots, a_{n}\right\rangle$ and $V \cong$ $\left[a_{1}, \ldots, a_{n}\right]$ relative to the basis $x_{1}, \ldots, x_{n}$.

If $L$ is binary with $\mathfrak{n} L=\alpha \mathcal{O}$, we denote by $a(L):=\operatorname{det} L \alpha^{-2}$ and by $R(L):=$ ord $\operatorname{vol} L-2 \operatorname{ord} \mathfrak{n} L=\operatorname{ord} a(L) . a(L) \in \dot{F} / \mathcal{O}^{\times 2}$ is an invariant of $L$ and it determines the class of $L$ up to scaling. If $L \cong \prec \alpha, \beta \succ$ then $a(L)=\frac{\beta}{\alpha}$.

We denote by $\mathcal{A}=\mathcal{A}_{F} \subset \dot{F} / \mathcal{O}^{\times 2}$ the set of all possible values of $a(L)$, where $L$ is an arbitrary binary lattice. We have $\mathcal{A}=\left\{a \in \frac{1}{4} \mathcal{O} \mid a \neq 0, \mathfrak{d}(-a) \subseteq \mathcal{O}\right\}$. If ord $a=R$ and $d(-a)=d$, then $a \in \frac{1}{4} \mathcal{O}$ means $R \geq-2 e$, while $\mathfrak{d}(-a) \subseteq \mathcal{O}$ means $R+d=\operatorname{ord} \mathfrak{d}(-a) \geq 0$.

If $a(L)=a=\pi^{R} \varepsilon$ with $d(-a)=d$ then:

$L$ is nonmodular, proper modular or improper modular iff $R>0, R=0$, resp. $R<0$.

If $R$ is odd then $R>0$.

The inequality $R+2 e \geq 0$ becomes equality iff $a \in-\frac{1}{4} \mathcal{O}^{\times 2}$ or $a \in-\frac{\Delta}{4} \mathcal{O}^{\times 2}$. We have $a(L)=-\frac{1}{4}$, resp. $a(L)=-\frac{\Delta}{4}$, when $L \cong \pi^{r} A(0,0)$, resp. $\pi^{r} A(2,2 \rho)$, for some integer $r$.

The inequality $R+d \geq 0$ becomes equality iff $a \in-\frac{\Delta}{4} \mathcal{O}^{\times 2}$.

A special type of BONG is the so-called "good BONG". If $L \cong \prec a_{1}, \ldots, a_{n} \succ$ relative to some BONG $x_{1}, \ldots, x_{n}$ and ord $a_{i}=R_{i}$ we say that the BONG $x_{1}, \ldots, x_{n}$ is good if $R_{i} \leq R_{i+2}$ for any $1 \leq i \leq n-2$.

Remark. The condition $R_{i} \leq R_{i+2}$ for $1 \leq i \leq n-2$ is equivalent to the condition that the sequence $\left(R_{i}+R_{i+1}\right)$ is increasing.

A set $x_{1}, \ldots, x_{n}$ of orthogonal vectors with $Q\left(x_{i}\right)=a_{i}$ and ord $a_{i}=R_{i}$ is a good BONG for some lattice iff $R_{i} \leq R_{i+2}$ for all $1 \leq i \leq n-2$ and $a_{i+1} / a_{i} \in \mathcal{A}$ for all $1 \leq i \leq n-1$. The condition $a_{i+1} / a_{i} \in \mathcal{A}$ is equivalent to $R_{i+1}-R_{i}+2 e \geq 0$ and $R_{i+1}-R_{i}+d\left(-a_{i} a_{i+1}\right) \geq 0$. As consequences of $a_{i+1} / a_{i} \in \mathcal{A}$, if $R_{i+1}-R_{i}$ is odd then it is positive, if $R_{i+1}-R_{i}=-2 e$ then $a_{i+1} / a_{i} \in-\frac{1}{4} \mathcal{O}^{\times 2}$ or $-\frac{\Delta}{4} \mathcal{O}^{\times 2}$ and if $R_{i+1}-R_{i}+d\left(-a_{i} a_{i+1}\right)=0$ then $a_{i+1} / a_{i} \in-\frac{\Delta}{4} \mathcal{O}^{\times 2}$.

The good BONGs enjoy some properties similar to those of orthogonal bases. If $L \cong \prec a_{1}, \ldots, a_{n} \succ$ relative to some good BONG $x_{1}, \ldots, x_{n}$ and ord $a_{i}=R_{i}$ then $L^{\sharp} \cong \prec a_{1}^{-1}, \ldots, a_{n}^{-1} \succ$ relative to the good BONG $x_{n}^{\sharp}, \ldots, x_{1}^{\sharp}$, where $x_{i}^{\sharp}=Q(x)^{-1} x_{i}$. Also if for some $1 \leq i \leq j \leq n$ we have $\prec x_{i}, \ldots, x_{j} \succ \cong \prec b_{i}, \ldots, b_{j} \succ$ relative to some other good BONG $y_{i}, \ldots, y_{j}$ then $L \cong \prec a_{1}, \ldots, a_{i-1}, b_{i}, \ldots, b_{j}, a_{i+1}, \ldots, a_{n} \succ$ relative to the good BONG $x_{1}, \ldots, x_{i-1}, y_{i}, \ldots, y_{j}, x_{i+1}, \ldots, x_{n}$. There are some differences though from the orthogonal bases. E.g. the relation $L=\prec x_{1}, \ldots, x_{i} \succ \perp$ $\prec x_{i+1}, \ldots, x_{n} \succ$ holds iff $R_{i} \leq R_{i+1}$.

The orders $R_{i}=$ ord $a_{i}$ are independent of the choice of the good BONGs and they are in 1-1 correspondence with the invariants $t, \operatorname{dim} L_{k}, \mathfrak{s}_{k}:=\mathfrak{s} L_{k}$ and $\mathfrak{n} L^{\mathfrak{s} k}$, 
where $L=L_{1} \perp \ldots \perp L_{t}$ is a Jordan splitting. More precisely, if $\mathfrak{s}_{k}=\mathfrak{p}^{r_{k}}$, $\mathfrak{n} L^{\mathfrak{s}_{k}}=\mathfrak{p}^{u_{k}}$ and $n_{k}=\sum_{l<k} \operatorname{dim} L_{l}$, then the sequence $R_{n_{k-1}+1}, \ldots, R_{n_{k}}$ is $r_{k}, \ldots, r_{k}$ if $L_{k}$ is proper (i.e. if $r_{k}=u_{k}$ ), and it is $u_{k}, 2 r_{k}-u_{k}, \ldots, u_{k}, 2 r_{k}-u_{k}$ otherwise; see [1, Lemma 4.7].

The good BONGs are closely connected with the maximal norm splittings. A splitting $L=L_{1} \perp \ldots \perp L_{t}$ is called a maximal norm splitting if $\mathfrak{s} L_{1} \supseteq \ldots \supseteq \mathfrak{s} L_{t}$ and $\operatorname{dim} L_{i} \leq 2, L_{i}$ is modular and $\mathfrak{n} L_{i}=\mathfrak{n} L^{\mathfrak{s} L_{i}}$ for all $1 \leq i \leq t$. Condition $\mathfrak{n} L_{i}=\mathfrak{n} L^{\mathfrak{s} L_{i}}$ is equivalent to $\mathfrak{n} L_{1} \supseteq \ldots \supseteq \mathfrak{n} L_{t}$ and $\mathfrak{n} L_{1}^{\sharp} \subseteq \ldots \subseteq \mathfrak{n} L_{t}^{\sharp}$. If we put together the BONGs of the components $L_{1}, \ldots, L_{t}$ of a maximal norm splitting we get a good BONG for $L$. Conversely any good BONG of a lattice can be obtained by putting together some BONGs of the components of some maximal norm splitting. Moreover, the splitting can be chosen such that all binary components are improper modular. An explicit algorithm for finding a maximal norm splitting and, hence, a good BONG of a lattice is provided in [2, Section 7].

\section{THE INVARIANTS $\alpha_{i}$}

Let $L$ be a lattice over the dyadic field $F$. Let $L \cong \prec a_{1}, \ldots, a_{n} \succ$ relative to a good BONG and let $R_{i}:=\operatorname{ord} a_{i}$. Also let $L=L_{1} \perp \ldots \perp L_{t}$ be a Jordan decomposition. We keep the notation of [4, $\mathfrak{s}_{k}:=\mathfrak{s} L_{k}, \mathfrak{g}_{k}:=\mathfrak{g} L^{\mathfrak{s}_{k}}, \mathfrak{w}_{k}:=\mathfrak{w} L^{\mathfrak{s}_{k}}$ but, in order to avoid confusion, we write $\mathrm{a}_{k}$ for O'Meara's $a_{k}$. Also we denote $r_{k}=\operatorname{ord} \mathfrak{s}_{k}, u_{k}=\operatorname{ord} \mathfrak{a}_{k}=\operatorname{ord} \mathfrak{n} L^{\mathfrak{s}_{k}}$. Associated to our splitting we have the Jordan chain $L_{(1)} \subset \ldots \subset L_{(t)}$ and the inverse Jordan chain $L_{(1)}^{*} \supset \ldots \supset L_{(t)}^{*}$, where $L_{(k)}:=L_{1} \perp \ldots \perp L_{k}$ and $L_{(k)}^{*}:=L_{k} \perp \ldots \perp L_{t}$.

Since the $R_{i}$ 's are invariants of $L$ we will write $R_{i}=R_{i}(L)$.

Definition 1. For any $1 \leq i \leq n-1$ we define $\alpha_{i}=\alpha_{i}(L)$ by:

$$
\begin{aligned}
\alpha_{i}:=\min \left(\left\{\left(R_{i+1}-R_{i}\right) / 2+e\right\} \cup\left\{R_{i+1}-R_{j}+d\left(-a_{j} a_{j+1}\right) \mid 1 \leq j \leq i\right\}\right. \\
\\
\left.\cup\left\{R_{j+1}-R_{i}+d\left(-a_{j} a_{j+1}\right) \mid i \leq j<n\right\}\right) .
\end{aligned}
$$

Apparently $\alpha_{i}(L)$ defined this way depends on the choice of the good BONG. We will show later that, in fact, it depends only on $L$. For the time being we will mean $\alpha_{i}(L)$ with respect to a given good BONG. We now give some properties of the $\alpha_{i}$ 's.

Lemma 2.1. If $k \leq i<l$ then, in the set defining $\alpha_{i}$, we can replace $\left(R_{i+1}-R_{i}\right) / 2+$ $e$ and all the terms corresponding to indices $k \leq j<l$ by $\alpha_{i-k+1}\left(\prec a_{k}, \ldots, a_{l} \succ\right)$. In particular, $\alpha_{i} \leq \alpha_{i-k+1}\left(\prec a_{k}, \ldots, a_{l} \succ\right)$.

Proof. By definition $\alpha_{i-k+1}\left(\prec a_{k}, \ldots, a_{l} \succ\right)=\min \left(\left\{\left(R_{i+1}-R_{i}\right) / 2+e\right\} \cup\left\{R_{i+1}-\right.\right.$ $\left.\left.R_{j}+d\left(-a_{j} a_{j+1}\right) \mid k \leq j \leq i\right\} \cup\left\{R_{j+1}-R_{i}+d\left(-a_{j} a_{j+1}\right) \mid i \leq j<l\right\}\right)$, hence the conclusion.

Lemma 2.2. The sequence $\left(R_{i}+\alpha_{i}\right)$ is increasing and the sequence $\left(-R_{i+1}+\alpha_{i}\right)$ is decreasing.

Proof. Let $1 \leq i \leq h \leq n-1$. We have $R_{i}+R_{i+1} \leq R_{h}+R_{h+1}$. From Definition 1 we get $R_{i}+\alpha_{i}=\min \left(\left\{\left(R_{i}+R_{i+1}\right) / 2+e\right\} \cup\left\{R_{i}+R_{i+1}-R_{j}+d\left(-a_{j} a_{j+1}\right) \mid 1 \leq j \leq\right.\right.$ $\left.i\} \cup\left\{R_{j+1}+d\left(-a_{j} a_{j+1}\right) \mid i \leq j<n\right\}\right)$ and $-R_{i+1}+\alpha_{i}=\min \left(\left\{-\left(R_{i}+R_{i+1}\right) / 2+e\right\} \cup\right.$ $\left.\left\{-R_{j}+d\left(-a_{j} a_{j+1}\right) \mid 1 \leq j \leq i\right\} \cup\left\{R_{j+1}-R_{i}-R_{i+1}+d\left(-a_{j} a_{j+1}\right) \mid i \leq j<n\right\}\right)$, and similarly for $R_{h}+\alpha_{h}$ and $-R_{h+1}+\alpha_{h}$. In order to prove that $R_{i}+\alpha_{i} \leq R_{h}+\alpha_{h}$ 
we show that the elements in the set that has $R_{i}+\alpha_{i}$ as its minimum are less than or equal to the corresponding elements for $R_{h}+\alpha_{h}$. The same holds for $-R_{i+1}+\alpha_{i} \geq-R_{h+1}+\alpha_{h}$.

The proof is straightforward and uses the fact that $R_{l}+R_{l+1}$ is an increasing sequence. For terms involving $d\left(-a_{j} a_{j+1}\right)$ we consider the cases $j \leq i, i \leq j \leq h$ and $h \leq j$ and use the inequalities among $R_{i}+R_{i+1}, R_{j}+R_{j+1}$ and $R_{h}+R_{h+1}$ that occur in each case.

Corollary 2.3. Suppose that $1 \leq i \leq j \leq n-1$ and $R_{i}+R_{i+1}=R_{j}+R_{j+1}$. Then:

(i) $R_{i}+\alpha_{i}=\ldots=R_{j}+\alpha_{j}$ and $-R_{i+1}+\alpha_{i}=\ldots=-R_{j+1}+\alpha_{j}$.

(ii) $R_{k}=R_{l}$ for any $k, l \in[i, j+1]$ of the same parity and $\alpha_{k}=\alpha_{l}$ for any $k, l \in[i, j]$ of the same parity.

(iii) If $\alpha_{k}=\left(R_{k+1}-R_{k}\right) / 2+e$ for some $i \leq k \leq j$ then $\alpha_{k}=\left(R_{k+1}-R_{k}\right) / 2+e$ for all $i \leq k \leq j$.

In the particular case when $j=i+1$ we get the following statement:

If $1 \leq i \leq n-2$ and $R_{i}=R_{i+2}$ then $R_{i}+\alpha_{i}=R_{i+1}+\alpha_{i+1},-R_{i+1}+\alpha_{i}=$ $-R_{i+2}+\alpha_{i+1}$ and $\alpha_{i}=\left(R_{i+1}-R_{i}\right) / 2+e$ is equivalent to $\alpha_{i+1}=\left(R_{i+2}-R_{i+1}\right) / 2+e$.

Proof. For (i) we note that $R_{i}+R_{i+1}=\left(R_{i}+\alpha_{i}\right)-\left(-R_{i+1}+\alpha_{i}\right)$ and $R_{j}+R_{j+1}=$ $\left(R_{j}+\alpha_{j}\right)-\left(-R_{j+1}+\alpha_{j}\right)$ and use Lemma 2.2. By using the fact that $R_{k}+R_{k+1}$ is an increasing sequence we get $R_{i}+R_{i+1}=R_{i+1}+R_{i+2}=\ldots=R_{j}+R_{j+1}$, which is equivalent to the first part of (ii). For the second part of (ii) use (i). Finally (iii) follows from $R_{i}+\alpha_{i}=\ldots=R_{j}+\alpha_{j}, R_{i}+R_{i+1}=\ldots=R_{j}+R_{j+1}$ and the fact that $\alpha_{k}=\left(R_{k+1}-R_{k}\right) / 2+e$ is equivalent to $R_{k}+\alpha_{k}=\left(R_{k}+R_{k+1}\right) / 2+e$.

Lemma 2.4. Suppose that $1 \leq i<n$ and $1 \leq k \leq h<l \leq n$. Then:

(i) If $h \leq i$ then all terms in the definition of $\alpha_{i}$ corresponding to indices $k \leq$ $j \leq h$ can be replaced by $R_{i+1}-R_{h+1}+\alpha_{h-k+1}\left(\prec a_{k}, \ldots, a_{l} \succ\right)$. In particular, all terms with $1 \leq j \leq h$ can be replaced by $R_{i+1}-R_{h+1}+\alpha_{h}$.

(ii) If $i \leq h$ then all terms in the definition of $\alpha_{i}$ corresponding to indices $h \leq j<l$ can be replaced by $R_{h}-R_{i}+\alpha_{h-k+1}\left(\prec a_{k}, \ldots, a_{l} \succ\right)$. In particular, all terms with $h \leq j<n$ can be replaced by $R_{h}-R_{i}+\alpha_{h}$.

Proof. By Lemma 2.1 we have $\alpha_{h-k+1}\left(\prec a_{k}, \ldots, a_{l} \succ\right) \geq \alpha_{h}$.

(i) By Lemma 2.2 we have $\alpha_{i} \leq R_{i+1}-R_{h+1}+\alpha_{h} \leq R_{i+1}-R_{h+1}+$ $\alpha_{h-k+1}\left(\prec a_{k}, \ldots, a_{l} \succ\right)$. If $k \leq j \leq h$ then $\alpha_{h-k+1}\left(\prec a_{k}, \ldots, a_{l} \succ\right) \leq R_{h+1}-R_{j}+$ $d\left(-a_{j} a_{j+1}\right)$ so $R_{i+1}-R_{h+1}+\alpha_{h-k+1}\left(\prec a_{k}, \ldots, a_{l} \succ\right) \leq R_{i+1}-R_{j}+d\left(-a_{j} a_{j+1}\right)$. Therefore if we add $R_{i+1}-R_{h+1}+\alpha_{h-k+1}\left(\prec a_{k}, \ldots, a_{l} \succ\right)$ to the set that defines $\alpha_{i}$ and remove any one of $R_{i+1}-R_{j}+d\left(-a_{j} a_{j+1}\right)$ with $k \leq j \leq h$ then $\alpha_{i}$ does not change.

(ii) By Lemma 2.2 we have $\alpha_{i} \leq R_{h}-R_{i}+\alpha_{h} \leq R_{h}-R_{i}+\alpha_{h-k+1}\left(\prec a_{k}, \ldots, a_{l} \succ\right)$. If $h \leq j<l$ then $\alpha_{h-k+1}\left(\prec a_{k}, \ldots, a_{l} \succ\right) \leq R_{j+1}-R_{h}+d\left(-a_{j} a_{j+1}\right)$ so $R_{h}-$ $R_{i}+\alpha_{h-k+1}\left(\prec a_{k}, \ldots, a_{l} \succ\right) \leq R_{j+1}-R_{i}+d\left(-a_{j} a_{j+1}\right)$. Thus if we add $R_{h}-$ $R_{i}+\alpha_{h-k+1}\left(\prec a_{k}, \ldots, a_{l} \succ\right)$ to the set that defines $\alpha_{i}$ and remove any one of $R_{j+1}-R_{i}+d\left(-a_{j} a_{j+1}\right)$ with $h \leq j<l$ then $\alpha_{i}$ does not change.

If we take $k=1$ and $l=n$ then $\alpha_{h-k+1}\left(\prec a_{k}, \ldots, a_{l} \succ\right)$ becomes $\alpha_{h}\left(\prec a_{1}, \ldots, a_{n} \succ\right)=\alpha_{h}(L)=\alpha_{h}$ so we get the second claims of (i) and (ii).

Corollary 2.5. For any $1 \leq i \leq n-1$ we have:

(i) $\alpha_{i}=\min \left\{\left(R_{i+1}-R_{i}\right) / 2+e, R_{i+1}-R_{i}+d\left(-a_{i} a_{i+1}\right), R_{i+1}-R_{i}+\alpha_{i-1}, R_{i+1}-\right.$ $\left.R_{i}+\alpha_{i+1}\right\}$. 
(ii) $\alpha_{i}=\min \left\{\left(R_{i+1}-R_{i}\right) / 2+e, R_{i+1}-R_{i}+d\left(-a_{i} a_{i+1}\right), R_{i+1}-R_{i}+\right.$ $\left.\alpha_{i-1}\left(\prec a_{1}, \ldots, a_{i} \succ\right), R_{i+1}-R_{i}+\alpha_{1}\left(\prec a_{i+1}, \ldots, a_{n} \succ\right)\right\}$.

(The terms that do not make sense, i.e. $R_{i+1}-R_{i}+\alpha_{i-1}$ and $R_{i+1}-R_{i}+$ $\alpha_{i-1}\left(\prec a_{1}, \ldots, a_{i} \succ\right)$ when $i=1$, or $R_{i+1}-R_{i}+\alpha_{i+1}$ and $R_{i+1}-R_{i}+$ $\alpha_{1}\left(\prec a_{i+1}, \ldots, a_{n} \succ\right)$ when $i=n-1$, are ignored.)

Proof. (i) By Lemma 2.4 (i), resp. (ii), in the set defining $\alpha_{i}, R_{i+1}-R_{i}+\alpha_{i-1}$ can replace all the terms $R_{i+1}-R_{j}+d\left(-a_{j} a_{j+1}\right)$ with $1 \leq j \leq i-1$, while $R_{i+1}-R_{i}+\alpha_{i+1}$ replaces all $R_{j+1}-R_{i}+d\left(-a_{j} a_{j+1}\right)$ with $i+1 \leq j<n$. Therefore $\alpha_{i}=\min \left\{\left(R_{i+1}-R_{i}\right) / 2+e, R_{i+1}-R_{i}+d\left(-a_{i} a_{i+1}\right), R_{i+1}-R_{i}+\alpha_{i-1}, R_{i+1}-R_{i}+\right.$ $\left.\alpha_{i+1}\right\}$.

(ii) Same as (i) but this time the terms corresponding to $1 \leq j \leq i-1$ are replaced by $R_{i+1}-R_{i}+\alpha_{i-1}\left(\prec a_{1}, \ldots, a_{i} \succ\right)$ and those corresponding to $i+1 \leq j<n$ by $R_{i+1}-R_{i}+\alpha_{1}\left(\prec a_{i+1}, \ldots, a_{n} \succ\right)$.

Remark 2.6. We have $L^{\sharp} \cong \prec a_{1}^{\sharp}, \ldots, a_{n}^{\sharp} \succ$ with $a_{i}^{\sharp}=a_{n+1-i}^{-1}$ and $R_{i}^{\sharp}:=\operatorname{ord} a_{i}^{\sharp}=$ $-R_{n+1-i}$. One can easily see that $\alpha_{i}^{\sharp}:=\alpha_{i}\left(L^{\sharp}\right)=\alpha_{n-i}$. Also, the $\alpha_{i}$ 's are invariant to scaling.

Lemma 2.7. If $1 \leq i \leq n-1$ then:

(i) $\alpha_{i} \geq 0$ with equality iff $R_{i+1}-R_{i}=-2 e$.

(ii) If $R_{i+1}-R_{i} \geq 2 e$ then $\alpha_{i}=\left(R_{i+1}-R_{i}\right) / 2+e$.

(iii) If $R_{i+1}-R_{i} \leq 2 e$ then $\alpha_{i} \geq R_{i+1}-R_{i}$ with equality iff $R_{i+1}-R_{i}=2 e$ or it is odd.

(iv) $\alpha_{i}$ is an odd integer unless $\alpha_{i}=\left(R_{i+1}-R_{i}\right) / 2+e$.

Proof. We use induction on $n$. For $n=1$ our lemma is vacuous.

For the induction step let $1 \leq i \leq n-1$ and let $L^{\prime}=\prec a_{1}, \ldots, a_{i} \succ$ and $L^{\prime \prime}=\prec a_{i+1}, \ldots, a_{n} \succ$. By Corollary 2.5(ii) we have $\alpha_{i}=\min \left\{\left(R_{i+1}-R_{i}\right) / 2+\right.$ $\left.e, R_{i+1}-R_{i}+d\left(-a_{i} a_{i+1}\right), R_{i+1}-R_{i}+\alpha, R_{i+1}-R_{i}+\beta\right\}$, where $\alpha=\alpha_{i-1}\left(L^{\prime}\right)$ and $\beta=\alpha_{1}\left(L^{\prime \prime}\right)$. (We ignore $\alpha$ and $\beta$ whenever they are not defined.) By the induction hypothesis $\alpha, \beta$ satisfy (i)-(iv) of the lemma.

We have $\left(R_{i+1}-R_{i}\right) / 2+e \geq 0$ with equality iff $R_{i+1}-R_{i}=-2 e$ and $R_{i+1}-R_{i}+$ $d\left(-a_{i} a_{i+1}\right) \geq 0$ with equality iff $a_{i+1} / a_{i} \in-\frac{\Delta}{4} \mathcal{O}^{\times^{2}}$, which implies $R_{i+1}-R_{i}=$ $-2 e$. If $R_{i+2}-R_{i+1}>2 e$ then $\beta=\left(R_{i+2}-R_{i+1}\right) / 2+e>2 e$ so $R_{i+1}-R_{i}+$ $\beta>R_{i+1}-R_{i}+2 e \geq 0$. Similarly with $R_{i+1}-R_{i}+\alpha$ if $R_{i}-R_{i-1}>2 e$. If $R_{i+2}-R_{i+1} \leq 2 e$ then, by the induction hypothesis, $\beta \geq R_{i+2}-R_{i+1}$ with equality iff $R_{i+2}-R_{i+1}$ is odd or it is $2 e$. Thus $R_{i+1}-R_{i}+\beta \geq R_{i+2}-R_{i} \geq 0$ with equality iff $R_{i}=R_{i+2}$ and $R_{i+2}-R_{i+1}$ is odd or $2 e$. Suppose this happens. If $R_{i+2}-R_{i+1}=2 e$ then $R_{i+1}-R_{i}=R_{i+1}-R_{i+2}=-2 e$. If $R_{i+2}-R_{i+1}$ is odd then so is $R_{i+1}-R_{i}=R_{i+1}-R_{i+2}$ so both must be positive. But this is impossible. Similar results hold for $R_{i+1}-R_{i}+\alpha$ when $R_{i}-R_{i-1} \leq 2 e$. Thus we have (i).

If $R_{i+1}-R_{i} \geq 2 e$ then $\alpha, \beta \geq 0$ so $R_{i+1}-R_{i}+d\left(-a_{i} a_{i+1}\right), R_{i+1}-R_{i}+\alpha, R_{i+1}-$ $R_{i}+\beta \geq R_{i+1}-R_{i} \geq\left(R_{i+1}-R_{i}\right) / 2+e$. Hence $\alpha_{i}=\left(R_{i+1}-R_{i}\right) / 2+e$ and we have (ii).

We now prove (iii). If $R_{i+1}-R_{i}=2 e$ then (ii) implies that $\alpha_{i}=\left(R_{i+1}-R_{i}\right) / 2+$ $e=2 e=R_{i+1}-R_{i}$ so we are done. If $R_{i+1}-R_{i}<2 e$ is odd then $d\left(-a_{i} a_{i+1}\right)=0$ and $\alpha, \beta \geq 0$ so $\alpha_{i}=\min \left\{\left(R_{i+1}-R_{i}\right) / 2+e, R_{i+1}-R_{i}\right\}=R_{i+1}-R_{i}$. Finally if $R_{i+1}-R_{i}<2 e$ is even then ord $a_{i} a_{i+1}=R_{i}+R_{i+1}$ is even so $d\left(-a_{i} a_{i+1}\right)>0$. Also $R_{i}-R_{i-1}, R_{i+2}-R_{i+1} \geq R_{i}-R_{i+1}>-2 e\left(R_{i-1} \leq R_{i+1}\right.$ and $\left.R_{i} \leq R_{i+2}\right)$ so by (i) 
$\alpha, \beta>0$. We have $R_{i+1}-R_{i}+d\left(-a_{1} a_{2}\right), R_{i+1}-R_{i}+\alpha, R_{i+1}-R_{i}+\beta>R_{i+1}-R_{i}$. Since also $\left(R_{i+1}-R_{i}\right) / 2+e>R_{i+1}-R_{i}$ (we have $R_{i+1}-R_{i}<2 e$ ) we get $\alpha_{i}>R_{i+1}-R_{i}$.

We now prove (iv). If $R_{i+1}-R_{i} \geq 2 e$ then (ii) implies $\alpha_{i}=\left(R_{i+1}-R_{i}\right) / 2+e$ so (iv) is vacuous. If $R_{i+1}-R_{i}<2 e$ is odd then (iii) implies $\alpha_{i}=R_{i+1}-R_{i}$ so $\alpha_{i}$ is odd. If $R_{i+1}-R_{i}<2 e$ is even then again ord $a_{i} a_{i+1}$ is even so $d\left(-a_{i} a_{i+1}\right)>0$. Suppose $\alpha_{i}<\left(R_{i+1}-R_{i}\right) / 2+e$. If $\alpha_{i}=R_{i+1}-R_{i}+d\left(-a_{i} a_{i+1}\right)$ then if $d\left(-a_{i} a_{i+1}\right)$ is odd $\alpha_{i}$ will also be odd so we are done. Otherwise $d\left(-a_{i} a_{i+1}\right)=2 e$ or $\infty$ so $\alpha_{i}=R_{i+1}-R_{i}+d\left(-a_{i} a_{i+1}\right) \geq R_{i+1}-R_{i}+2 e \geq\left(R_{i+1}-R_{i}\right) / 2+e>\alpha_{i}$. (We have $R_{i+1}-R_{i}+2 e \geq 0$.) Contradiction. If $\alpha_{i}=R_{i+1}-R_{i}+\alpha$ then $\alpha_{i}$ is odd unless $\alpha$ is not odd, which would imply $\alpha=\left(R_{i}-R_{i-1}\right) / 2+e$. So $\alpha_{i}=R_{i+1}-R_{i}+\left(R_{i}-R_{i-1}\right) / 2+e \geq\left(R_{i+1}-R_{i}\right) / 2+e>\alpha_{i}$. (We have $R_{i+1} \geq R_{i-1}$.) Contradiction. Similar results hold if $\alpha_{i}=R_{i+1}-R_{i}+\beta$ since $R_{i+1}-R_{i}+\left(R_{i+2}-\right.$ $\left.R_{i+1}\right) / 2+e \geq\left(R_{i+1}-R_{i}\right) / 2+e>\alpha_{i}$. (We have $R_{i+2} \geq R_{i}$.)

Corollary 2.8. (i) $\alpha_{i} \in \mathbb{Z}$ except when $R_{i+1}-R_{i}$ is odd and $>2 e$.

(ii) $\alpha_{i}$ is $<2 e,=2 e$ or $>2 e$ if $R_{i+1}-R_{i}$ is $<2 e,=2 e$ or $>2 e$ accordingly.

(iii) $\alpha_{i} \in([0,2 e] \cap \mathbb{Z}) \cup\left((2 e, \infty) \cap \frac{1}{2} \mathbb{Z}\right)$.

Proof. (i) If $R_{i+1}-R_{i}>2 e$ then $\alpha_{i}=\left(R_{i+1}-R_{i}\right) / 2+e$. If $R_{i+1}-R_{i}$ is even then $\alpha_{i} \in \mathbb{Z}$, while if it is odd then $\alpha_{i} \in \frac{1}{2} \mathbb{Z} \backslash \mathbb{Z}$. Suppose now that $R_{i+1}-R_{i} \leq 2 e$. If $R_{i+1}-R_{i}$ is odd then $\alpha_{i}=R_{i+1}-R_{i} \in \mathbb{Z}$. If $R_{i+1}-R_{i}$ is even then either $\alpha_{i}$ is an odd integer or $\alpha_{i}=\left(R_{i+1}-R_{i}\right) / 2+e \in \mathbb{Z}$.

(ii) If $R_{i+1}-R_{i}<2 e$ then $\alpha_{i} \leq\left(R_{i+1}-R_{i}\right) / 2+e<2 e$. If $R_{i+1}-R_{i}=2 e$ then $\alpha_{i}=\left(R_{i+1}-R_{i}\right) / 2+e=2 e$. If $R_{i+1}-R_{i}>2 e$ then $\alpha_{i}=\left(R_{i+1}-R_{i}\right) / 2+e>2 e$.

(iii) We have $\alpha_{i} \geq 0$. If $\alpha_{i} \leq 2 e$ then $R_{i+1}-R_{i} \leq 2 e$ so $\alpha_{i} \in \mathbb{Z}$. If $\alpha_{i}>2 e$ then $R_{i+1}-R_{i}>2 e$ so $\alpha_{i}=\left(R_{i+1}-R_{i}\right) / 2+e \in(2 e, \infty) \cap \frac{1}{2} \mathbb{Z}$.

Corollary 2.9. In each of the following cases, $\alpha_{i}$ depends only on $R_{i+1}-R_{i}$ :

(i) If $R_{i+1}-R_{i} \geq 2 e$ or $R_{i+1}-R_{i} \in\{-2 e, 2-2 e, 2 e-2\}$ then $\alpha_{i}=\left(R_{i+1}-\right.$ $\left.R_{i}\right) / 2+e$.

(ii) If $R_{i+1}-R_{i}$ is odd, then $\alpha_{i}=\min \left\{\left(R_{i+1}-R_{i}\right) / 2+e, R_{i+1}-R_{i}\right\}$.

Proof. (i) If $R_{i+1}-R_{i} \geq 2 e$ then $\alpha_{i}=\left(R_{i+1}-R_{i}\right) / 2+e$ by Lemma 2.7(ii). If $R_{i+1}-R_{i}=-2 e$ then $\alpha_{i}=0=\left(R_{i+1}-R_{i}\right) / 2+e$. If $R_{i+1}-R_{i}=2-2 e$ then $\alpha_{i} \in \mathbb{Z}$ and $0<\alpha_{i} \leq\left(R_{i+1}-R_{i}\right) / 2+e=1$ so $\alpha_{i}=1=\left(R_{i+1}-R_{i}\right) / 2+e$. If $R_{i+1}-R_{i}=2 e-2$ then $\alpha_{i} \in \mathbb{Z}$ and $2 e-2=R_{i+1}-R_{i}<\alpha_{i} \leq\left(R_{i+1}-R_{i}\right) / 2+e=$ $2 e-1$ so $\alpha_{i}=2 e-1=\left(R_{i+1}-R_{i}\right) / 2+e$.

(ii) We use Lemma 2.7(ii) and (iii). If $R_{i+1}-R_{i}>2 e$ then $\alpha_{i}=\left(R_{i+1}-R_{i}\right) / 2+$ $e<R_{i+1}-R_{i}$. If $R_{i+1}-R_{i}<2 e$ then $\alpha_{i}=R_{i+1}-R_{i}<\left(R_{i+1}-R_{i}\right) / 2+e$. In both cases $\alpha_{i}=\min \left\{\left(R_{i+1}-R_{i}\right) / 2+e, R_{i+1}-R_{i}\right\}$.

Lemma 2.10. Let a be a norm generator of a lattice $L$ and let $\mathfrak{w} \supseteq 2 \mathfrak{s} L$ be a fractional ideal. Then $\mathfrak{w}=\mathfrak{w} L$ iff $\mathfrak{g} L=\mathrm{a} \mathcal{O}^{2}+\mathfrak{w}$ and we have either $\mathfrak{w}=2 \mathfrak{s} L$ or ord $\mathrm{a}+$ ord $\mathfrak{w}$ is odd.

Proof. For the necessity, see [4, 93A]. For the sufficiency it is enough to prove that, given another fractional ideal $\mathfrak{w}^{\prime}$ satisfying the hypothesis of the lemma, we have $\mathfrak{w}=\mathfrak{w}^{\prime}$. Suppose that $\mathfrak{w} \neq \mathfrak{w}^{\prime}$. We may assume that $\mathfrak{w} \supset \mathfrak{w}^{\prime}$. Since $\mathfrak{w} \supset \mathfrak{w}^{\prime} \supseteq 2 \mathfrak{s} L$ we must have that ord $a+$ ord $\mathfrak{w}$ is odd. Let $\mathfrak{w}=b \mathcal{O}$. Then $a+b \in a \mathcal{O}^{2}+\mathfrak{w}=$ $\mathfrak{g} L=\mathrm{a} \mathcal{O}^{2}+\mathfrak{w}^{\prime}$. So $\mathrm{a}+\mathrm{b}=\mathrm{a} \alpha^{2}+\mathrm{b}^{\prime}$ for some $\alpha \in \mathcal{O}$ and $\mathrm{b}^{\prime} \in \mathfrak{w}^{\prime} \subset \mathfrak{w}$. It follows that $1+\mathrm{b} / \mathrm{a}=\alpha^{2}+\mathrm{b}^{\prime} / \mathrm{a}$, which implies that $\mathfrak{d}(1+\mathrm{b} / \mathrm{a}) \subseteq \mathrm{b}^{\prime} / \mathrm{aO} \subset \mathrm{a}^{-1} \mathfrak{w}$. 
On the other hand ord $\mathrm{b} / \mathrm{a}=$ ord $\mathrm{a}^{-1} \mathfrak{w}$ is odd and, since $\mathrm{b} \mathcal{O}=\mathfrak{w} \subseteq \mathfrak{g} L \subseteq \mathrm{aO}$ and $\mathrm{b} \mathcal{O}=\mathfrak{w} \supset 2 \mathfrak{s} L \supseteq 4 \mathrm{a} \mathcal{O}$, we have $4 \mathcal{O} \subset \mathrm{b} / \mathrm{aO} \subseteq \mathcal{O}$. By [4, 63:5] we get $\mathfrak{d}(1+\mathrm{b} / \mathrm{a})=\mathrm{b} / \mathrm{a} \mathcal{O}=\mathrm{a}^{-1} \mathfrak{w}$, a contradiction.

Lemma 2.11. Let $J_{1}, \ldots, J_{s}$ be lattices in the same quadratic space and let $J=$ $\sum J_{k}$. If $\mathrm{a}_{k}$ and a are norm generators for $J_{k}$ and a and $J$, respectively, then:

$$
\mathfrak{g} J=\sum \mathfrak{g} J_{k}+2 \mathfrak{s} J \text { and } \mathfrak{w} J=\sum \mathfrak{w} J_{k}+\sum \mathfrak{a}^{-1} \mathfrak{d}\left(\mathrm{aa}_{k}\right)+2 \mathfrak{s} J .
$$

Proof. We have $\mathfrak{g} J_{k} \subseteq \mathfrak{g} J$ and $2 \mathfrak{s} J \subseteq \mathfrak{g} J$ so $\mathfrak{g} J \supseteq \sum \mathfrak{g} J_{k}+2 \mathfrak{s} J$. For the reverse inclusion note that $Q(J) \subseteq \sum Q\left(J_{k}\right)+2 \mathfrak{s} J$. Thus $\mathfrak{g} J=Q(J)+2 \mathfrak{s} J \subseteq \sum\left(Q\left(J_{k}\right)+\right.$ $\left.2 \mathfrak{s} J_{k}\right)+2 \mathfrak{s} J=\sum \mathfrak{g} J_{k}+2 \mathfrak{s} J$.

We have $a \mathcal{O}^{2} \subseteq \mathfrak{g} J$ and $2 \mathrm{a} \mathcal{O}=2 \mathfrak{n} J \subseteq 2 \mathfrak{s} J \subseteq \mathfrak{g} J$ so $\mathfrak{g} J=\mathrm{aO}^{2}+2 \mathrm{aO}+\mathfrak{g} J=$ $\mathrm{a} \mathcal{O}^{2}+2 \mathrm{a} \mathcal{O}+\sum \mathfrak{g} J_{k}+2 \mathfrak{s} J=\mathrm{a} \mathcal{O}^{2}+2 \mathrm{a} \mathcal{O}+\sum \mathrm{a}_{k} \mathcal{O}^{2}+\sum \mathfrak{w} J_{k}+2 \mathfrak{s} J$. But aO $\mathcal{O}^{2}+\sum \mathrm{a}_{k} \mathcal{O}^{2}+$ $2 \mathrm{aO}=\mathfrak{g}\left(\left\langle a, a_{1}, \ldots, \mathrm{a}_{s}\right\rangle\right)$. (We have $\mathfrak{s}\left(\left\langle a, a_{1}, \ldots, \mathrm{a}_{s}\right\rangle\right)=\mathfrak{n}\left(\left\langle a, a_{1}, \ldots, a_{s}\right\rangle\right)=a \mathcal{O}$.) But $\mathfrak{w}\left(\left\langle a, a_{1}, \ldots, a_{s}\right\rangle\right)=\sum a^{-1} \mathfrak{d}\left(a_{k}\right)+2 a \mathcal{O}$. (See [4, p. 280]. We have $a \mathfrak{d}\left(a_{k} / a\right)=$ $\left.\mathrm{a}^{-1} \mathfrak{d}\left(\mathrm{aa}_{k}\right).\right) \quad$ So $\mathfrak{g} J=\mathfrak{g}\left(\left\langle\mathrm{a}, \mathrm{a}_{1}, \ldots, \mathrm{a}_{s}\right\rangle\right)+\sum \mathfrak{w} J_{k}+2 \mathfrak{s} J=\mathfrak{a} \mathcal{O}^{2}+\sum \mathrm{a}^{-1} \mathfrak{d}\left(\mathrm{aa}_{k}\right)+$ $2 \mathrm{a} \mathcal{O}+\sum \mathfrak{w} J_{k}+2 \mathfrak{s} J=\mathrm{aO}^{2}+\sum \mathfrak{w} J_{k}+\sum \mathrm{a}^{-1} \mathfrak{d}\left(\mathrm{aa}_{k}\right)+2 \mathfrak{s} J$. (Recall, $2 \mathrm{a} \mathcal{O} \subseteq 2 \mathfrak{s} J$.) Let $\mathfrak{w}=\sum \mathfrak{w} J_{k}+\sum \mathrm{a}^{-1} \mathfrak{d}\left(\mathrm{aa}_{k}\right)+2 \mathfrak{s} J$. We have $\mathfrak{g} J=\mathfrak{a O}^{2}+\mathfrak{w}$ and $2 \mathfrak{s} J \subseteq \mathfrak{w}$. By Lemma 2.10 in order to prove that $\mathfrak{w}=\mathfrak{w} J$ we still need to prove that $\mathfrak{w}=2 \mathfrak{s} J$ or ord $a+$ ord $\mathfrak{w}$ is odd. If $\mathfrak{w} \neq 2 \mathfrak{s} J$, i.e. $\mathfrak{w} \supset 2 \mathfrak{s} J$, then $\mathfrak{w}=\mathfrak{a}^{-1} \mathfrak{d}\left(\mathrm{aa}_{k}\right)$ or $\mathfrak{w}=\mathfrak{w} J_{k}$ for some $k$. Suppose that $\mathfrak{w}=\mathfrak{w} J_{k}$. We cannot have $\mathfrak{w} J_{k}=2 \mathfrak{s} J_{k} \subseteq 2 \mathfrak{s} J$. So ord $a_{k}+\operatorname{ord} \mathfrak{w} J_{k}$ is odd, which implies that ord $a+\operatorname{ord} \mathfrak{w} J_{k}$ is odd unless ord ( $\operatorname{aa}_{k}$ ) is odd. But this would imply that $\mathrm{a}_{k} \mathcal{O}=\mathrm{a}^{-1} \mathfrak{d}\left(\mathrm{aa}_{k}\right) \subseteq \mathfrak{w}=\mathfrak{w} J_{k}$, so $\mathfrak{w} J_{k}=\mathrm{a}_{k} \mathcal{O}$, which contradicts the fact that ord $a_{k}+\operatorname{ord} \mathfrak{w} J_{k}$ is odd. Finally if $\mathfrak{w}=\mathfrak{a}^{-1} \mathfrak{d}\left(\operatorname{aa}_{k}\right)$ then ord $\mathrm{a}+\operatorname{ord} \mathfrak{w}=\operatorname{ord} \mathfrak{d}\left(\mathrm{aa}_{k}\right)$ is odd unless $\mathrm{aa}_{k} \in \Delta \dot{F}^{2}$. (If $\alpha \in \dot{F}$ has odd order then $\mathfrak{d}(\alpha)=\alpha \mathcal{O}$ has odd order. If ord $\alpha$ is even then ord $\mathfrak{d}(\alpha)=\operatorname{ord} \alpha+d(\alpha) \equiv$ $d(\alpha)(\bmod 2)$ is even iff $d(\alpha)=2 e$, i.e. iff $\alpha \in \Delta \dot{F}^{2}$.) But this implies that $\mathfrak{d}\left(\mathrm{aa}_{k}\right)=4 \mathrm{aa}_{k} \mathcal{O}$, i.e. $\mathfrak{w}=\mathrm{a}^{-1} \mathfrak{d}\left(\mathrm{aa}_{k}\right)=4 \mathrm{a}_{k} \mathcal{O} \subset 2 \mathfrak{s} J$, a contradiction.

Lemma 2.12. Suppose that $\mathfrak{n} L_{k}=\mathfrak{n} L^{\mathfrak{s}_{k}}, \mathfrak{n} L_{k+1}=\mathfrak{n} L^{\mathfrak{s}_{k+1}}$ and $\mathrm{a}_{k}$ and $\mathrm{a}_{k+1}$ are norm generators for $L_{k}$ and $L_{k+1}$, respectively. If $u_{k}+u_{k+1}$ is even, then

$$
\mathfrak{f}_{k}=\mathfrak{s}_{k}^{-2} \mathfrak{d}\left(\mathrm{a}_{k} \mathrm{a}_{k+1}\right)+\mathrm{a}_{k} \mathfrak{s}_{k}^{-2} \mathfrak{w} L_{(k+1)}^{*}+\mathrm{a}_{k+1} \mathfrak{w} L_{(k)}^{\sharp}+2 \mathfrak{p}^{\left(u_{k}+u_{k+1}\right) / 2-r_{k}} .
$$

Proof. We have $L^{\mathfrak{s}_{k}}=\mathfrak{s}_{k} L_{(k)}^{\sharp} \perp L_{(k+1)}^{*}$ and $L^{\mathfrak{s}_{k+1}}=\mathfrak{s}_{k+1} L_{(k)}^{\sharp} \perp L_{(k+1)}^{*}$. Now $L_{k+1} \subseteq L_{k+1}^{*} \subseteq L^{\mathfrak{s}_{k+1}}$ and $L_{k} \subseteq \mathfrak{s}_{k} L_{(k)}^{\sharp} \subseteq L^{\mathfrak{s}_{k}}$. Thus $\mathrm{a}_{k+1}$ is a norm generator for $L_{(k+1)}^{*}$ and for $L^{\mathfrak{s}_{k+1}}$ and $\mathrm{a}_{k}$ is a norm generator for $\mathfrak{s}_{k} L_{(k)}^{\sharp}$ and for $L^{\mathfrak{s}_{k}}$. Also $\pi^{2\left(r_{k+1}-r_{k}\right)} \mathrm{a}_{k}$ is a norm generator for $\mathfrak{s}_{k+1} L_{(k)}^{\sharp}$. By Lemma 2.11 we get $\mathfrak{w}_{k}=$ $\mathrm{a}_{k}^{-1} \mathfrak{d}\left(\mathrm{a}_{k} \mathrm{a}_{k+1}\right)+\mathfrak{w}\left(\mathfrak{s}_{k} L_{(k)}^{\sharp}\right)+\mathfrak{w} L_{(k+1)}^{*}+2 \mathfrak{s}_{k}=\mathrm{a}_{k}^{-1} \mathfrak{d}\left(\mathrm{a}_{k} \mathrm{a}_{k+1}\right)+\mathfrak{s}_{k}^{2} \mathfrak{w} L_{(k)}^{\sharp}+\mathfrak{w} L_{(k+1)}^{*}+$ $2 \mathfrak{s}_{k}$ and $\mathfrak{w}_{k+1}=\mathrm{a}_{k+1}^{-1} \mathfrak{d}\left(\pi^{2\left(r_{k+1}-r_{k}\right)} \mathrm{a}_{k} \mathrm{a}_{k+1}\right)+\mathfrak{w}\left(\mathfrak{s}_{k+1} L_{(k)}^{\sharp}\right)+\mathfrak{w} L_{(k+1)}^{*}+2 \mathfrak{s}_{k+1}=$ $\mathrm{a}_{k+1}^{-1} \mathfrak{s}_{k+1}^{2} \mathfrak{s}_{k}^{-2} \mathfrak{d}\left(\mathrm{a}_{k} \mathrm{a}_{k+1}\right)+\mathfrak{s}_{k+1}^{2} \mathfrak{w} L_{(k)}^{\sharp}+\mathfrak{w} L_{(k+1)}^{*}+2 \mathfrak{s}_{k+1}$.

By [4, 93:26] we have $\mathfrak{s}_{k}^{2} \mathfrak{f}_{k}=\mathfrak{d}\left(\mathrm{a}_{k} \mathrm{a}_{k+1}\right)+\mathrm{a}_{k+1} \mathfrak{w}_{k}+\mathrm{a}_{k} \mathfrak{w}_{k+1}+2 \mathfrak{p}^{\left(u_{k}+u_{k+1}\right) / 2+r_{k}}=$ $\mathfrak{d}\left(\mathrm{a}_{k} \mathrm{a}_{k+1}\right)+\mathrm{a}_{k}^{-1} \mathrm{a}_{k+1} \mathfrak{d}\left(\mathrm{a}_{k} \mathrm{a}_{k+1}\right)+\mathrm{a}_{k+1} \mathfrak{s}_{k}^{2} \mathfrak{w} L_{(k)}^{\sharp}+\mathrm{a}_{k+1} \mathfrak{w} L_{(k+1)}^{*}+2 \mathrm{a}_{k+1} \mathfrak{s}_{k}+$ $\mathrm{a}_{k} \mathrm{a}_{k+1}^{-1} \mathfrak{s}_{k+1}^{2} \mathfrak{s}_{k}^{-2} \mathfrak{d}\left(\mathrm{a}_{k} \mathrm{a}_{k+1}\right)+\mathrm{a}_{k} \mathfrak{s}_{k+1}^{2} \mathfrak{w} L_{(k)}^{\sharp}+\mathrm{a}_{k} \mathfrak{w} L_{(k+1)}^{*}+2 \mathrm{a}_{k} \mathfrak{s}_{k+1}+2 \mathfrak{p}^{\left(u_{k}+u_{k+1}\right) / 2+r_{k}}$.

But $\mathrm{a}_{k} \mathcal{O} \supseteq \mathrm{a}_{k+1} \mathcal{O}$ and $\mathrm{a}_{k} \mathfrak{s}_{k}^{-2} \subseteq \mathrm{a}_{k+1} \mathfrak{s}_{k+1}^{-2}$ ([4, 93:25]), so $u_{k} \leq u_{k+1}$ and $u_{k}-2 r_{k} \geq u_{k+1}-2 r_{k+1}$. Thus $\mathrm{a}_{k}^{-1} \mathrm{a}_{k+1} \mathfrak{d}\left(\mathrm{a}_{k} \mathrm{a}_{k+1}\right), \mathrm{a}_{k} \mathrm{a}_{k+1}^{-1} \mathfrak{s}_{k+1}^{2} \mathfrak{s}_{k}^{-2} \mathfrak{d}\left(\mathrm{a}_{k} \mathrm{a}_{k+1}\right) \subseteq$ $\mathfrak{d}\left(\mathrm{a}_{k} \mathrm{a}_{k+1}\right)$. Also $\mathrm{a}_{k} \mathfrak{s}_{k+1}^{2} \mathfrak{w} L_{(k)}^{\sharp} \subseteq \mathrm{a}_{k+1} \mathfrak{s}_{k}^{2} \mathfrak{w} L_{(k)}^{\sharp}$ and $\mathrm{a}_{k+1} \mathfrak{w} L_{(k+1)}^{*} \subseteq \mathrm{a}_{k} \mathfrak{w} L_{(k+1)}^{*}$. 
Also ord $a_{k+1} \mathfrak{s}_{k}=u_{k+1}+r_{k} \geq\left(u_{k}+u_{k+1}\right) / 2+r_{k}$ (we have $u_{k+1} \geq u_{k}$ ) and ord $a_{k} \mathfrak{s}_{k+1}=u_{k}+r_{k+1} \geq\left(u_{k}+u_{k+1}\right) / 2+r_{k}$ (we have $\left.u_{k}-2 r_{k} \geq u_{k+1}-2 r_{k+1}\right)$. Hence $2 \mathrm{a}_{k+1} \mathfrak{s}_{k}, 2 \mathrm{a}_{k} \mathfrak{s}_{k+1} \subseteq 2 \mathfrak{p}^{\left(u_{k}+u_{k+1}\right) / 2+r_{k}}$.

By removing all unnecessary terms (which are included in others) we get $\mathfrak{s}_{k}^{2} \mathfrak{f}_{k}=$ $\mathfrak{d}\left(\mathrm{a}_{k} \mathrm{a}_{k+1}\right)+\mathrm{a}_{k+1} \mathfrak{s}_{k}^{2} \mathfrak{w} L_{(k)}^{\sharp}+\mathrm{a}_{k} \mathfrak{w} L_{(k+1)}^{*}+2 \mathfrak{p}^{\left(u_{k}+u_{k+1}\right) / 2+r_{k}}$. When we divide by $\mathfrak{s}_{k}^{2}$ we get the desired result.

Suppose $L \cong \prec a_{1}, \ldots, a_{n} \succ$ relative to the good BONG $x_{1}, \ldots, x_{n}$. Let $L=$ $L^{1} \perp \ldots \perp L^{m}$ be a maximal norm splitting with all the binary components improper such that $x_{1}, \ldots, x_{n}$ is obtained by putting together the BONGs of $L^{1}, \ldots, L^{m}$. We choose the Jordan decomposition $L=L_{1} \perp \ldots \perp L_{t}$ with components obtained by putting together the $L^{j}$ 's of the same scale (see also the proof of 1. Lemma 4.7]). So the $L^{j}$ 's with $\mathfrak{s} L^{j}=\mathfrak{s}_{k}$ make a maximal norm splitting for $L_{k}$, those with $\mathfrak{s} L^{j} \supseteq \mathfrak{s}_{k}$ a maximal norm splitting for $L_{(k)}$ and those with $\mathfrak{s} L^{j} \subset \mathfrak{s}_{k}$ a maximal norm splitting for $L_{(k+1)}^{*}$. By putting together the BONGs of the components of these maximal norm splittings we get good BONGs for $L_{k}, L_{(k)}$ and $L_{(k+1)}^{*}$. It follows that $L_{k}=\prec x_{n_{k-1}+1}, \ldots, x_{n_{k}} \succ, L_{(k)}=\prec x_{1}, \ldots, x_{n_{k}} \succ$ and $L_{(k+1)}^{*}=$ $\prec x_{n_{k}+1}, \ldots, x_{n} \succ$. Also $\mathfrak{n} L_{k}=\mathfrak{n} L^{\mathfrak{s}_{k}}$. (For any $L^{j}$ with $\mathfrak{s} L^{j}=\mathfrak{s}_{k}$ we have $L^{j} \subseteq L_{k} \subseteq L^{\mathfrak{s}_{k}}$ and $\mathfrak{n} L^{j}=\mathfrak{n} L^{\mathfrak{s} L^{j}}=\mathfrak{n} L^{\mathfrak{s}_{k}}$.)

Lemma 2.13. (i) For any $n_{k-1}+1 \leq i \leq n_{k}$ we have $R_{i}=u_{k}$ if $i \equiv n_{k-1}+$ $1(\bmod 2)$ and $R_{i}=2 r_{k}-u_{k}$ if $i \equiv n_{k-1}(\bmod 2)$.

(ii) For any $n_{k-1}+1 \leq i \leq n_{k}$ we have $R_{i}=u_{k}$ if $i \equiv n_{k}+1(\bmod 2)$ and $R_{i}=2 r_{k}-u_{k}$ if $i \equiv n_{k}(\bmod 2)$.

(iii) $\pm a_{n_{k-1}+1}$ and $\pm \pi^{2 u_{k}-2 r_{k}} a_{n_{k}}$ are norm generators for $L_{k}$ and for $L^{\mathfrak{s}_{k}}$.

Proof. If $L_{k}$ is improper then $\operatorname{dim} L_{k}$ is even so $n_{k-1} \equiv n_{k}(\bmod 2)$. Also the sequence $R_{n_{k-1}+1}, \ldots, R_{n_{k}}$ is $u_{k}, 2 r_{k}-u_{k}, \ldots, u_{k}, 2 r_{k}-u_{k}$ so we get both (i) and (ii). If $L_{k}$ is proper then $u_{k}=r_{k}$ and the sequence $R_{n_{k-1}+1}, \ldots, R_{n_{k}}$ is $r_{k}, \ldots, r_{k}$. But $u_{k}=r_{k}$, so $r_{k}=u_{k}=2 r_{k}-u_{k}$ and again we get both (i) and (ii).

(iii) We have $L_{k} \cong \prec a_{n_{k-1}+1}, \ldots, a_{n_{k}} \succ$ so $a_{n_{k-1}+1}$ is a norm generator for $L_{k}$. We have $L_{k}^{\sharp} \cong \prec a_{n_{k}}^{-1}, \ldots, a_{n_{k-1}}^{-1} \succ$ so $a_{n_{k}}^{-1}$ is a norm generator for $L_{k}^{\sharp}=$ $\mathfrak{p}^{-r_{k}} L_{k}$. Therefore $\pi^{2 r_{k}} a_{n_{k}}^{-1}$ is a norm generator for $L_{k}$. But ord $a_{n_{k}}=2 r_{k}-u_{k}$, so $\pi^{2 u_{k}-4 r_{k}} a_{n_{k}}$ differs from $a_{n_{k}}^{-1}$ by the square of a unit. Since $\pi^{2 r_{k}} a_{n_{k}}^{-1}$ is a norm generator for $L_{k}$ so is $\pi^{2 r_{k}} \pi^{2 u_{k}-4 r_{k}} a_{n_{k}}=\pi^{2 u_{k}-2 r_{k}} a_{n_{k}}$. Since $\mathfrak{g} L_{k}$ is an additive group, $-a_{n_{k-1}+1}$ and $-\pi^{2 u_{k}-2 r_{k}} a_{n_{k}}$ will also be norm generators for $L_{k}$. We have $L_{k} \subseteq L^{\mathfrak{s}_{k}}$ and $\mathfrak{n} L_{k}=\mathfrak{n} L^{\mathfrak{s}_{k}}$ so $\pm a_{n_{k-1}+1}$ and $\pm \pi^{2 u_{k}-2 r_{k}} a_{n_{k}}$ are norm generators for $L^{\mathfrak{s} L_{k}}$ as well.

We now want to find relations between the $\alpha_{i}$ 's and the O'Meara invariants $\mathfrak{w}_{k}$ and $\mathfrak{f}_{k}$. In particular, this will prove that the $\alpha_{i}$ 's are invariants of the lattice $L$, i.e. they do not depend on the choice of the BONG of $L$.

Lemma 2.14. ord $\mathfrak{w} L=\min \left\{R_{1}+\alpha_{1}, R_{1}+e\right\}$. (If $n=1$ we ignore $R_{1}+\alpha_{1}$.)

If moreover $L_{1}$ is not unary then ord $\mathfrak{w} L=R_{1}+\alpha_{1}$.

Proof. Note that if $L_{1}$ is not unary, in particular if $L^{1}$ is binary, then $R_{1}=u_{1} \geq$ $2 r_{1}-u_{1}=R_{2}$ so $\alpha_{1} \leq\left(R_{2}-R_{1}\right) / 2+e \leq e$. Hence $\min \left\{R_{1}+\alpha_{1}, R_{1}+e\right\}=R_{1}+\alpha_{1}$ and so the two statements of the lemma are equivalent.

We use induction on $m$, the number of components in the maximal norm splitting we fixed for $L$. Suppose first that $m=1$. If $L=L^{1}$ is unary then $\mathfrak{w} L=2 \mathfrak{s} L=2 \mathfrak{p}^{R_{1}}$ 
so $\operatorname{ord} \mathfrak{w} L^{1}=R_{1}+e$, as claimed. If $L=L^{1}$ is binary and so improper modular then we may assume that it is unimodular since the statement is invariant upon scaling. Hence $R_{1}+R_{2}=0$ and $R_{1}=\operatorname{ord} \mathfrak{n} L>\operatorname{ord} \mathfrak{s} L=0$. Now $a_{1} \in Q(L)$ is a norm generator. Thus by [4, 93:10] there is $b \in \mathfrak{w} L$ such that $L \cong A\left(a_{1}, b\right)$. Also if $\mathfrak{w} L \supset 2 \mathfrak{s} L=2 \mathcal{O}$ then $\mathfrak{w} L=b \mathcal{O}$. Suppose first that $\mathfrak{w} L=2 \mathcal{O}$. Then $b \in 2 \mathcal{O}$ so ord $b \geq e$. Thus $d\left(-a_{1} a_{2}\right)=d(-\operatorname{det} L)=d\left(1-a_{1} b\right) \geq \operatorname{ord} a_{1} b \geq R_{1}+e$ so $R_{2}-R_{1}+d\left(-a_{1} a_{2}\right)=-2 R_{1}+d\left(-a_{1} a_{2}\right) \geq-R_{1}+e$. On the other hand $\left(R_{2}-R_{1}\right) / 2+$ $e=-R_{1}+e$ so $\alpha_{1}=\min \left\{\left(R_{2}-R_{1}\right) / 2+e, R_{2}-R_{1}+d\left(-a_{1} a_{2}\right)\right\}=-R_{1}+e$. Thus ord $\mathfrak{w} L=e=R_{1}+\alpha_{1}$. If $\mathfrak{w} L \supset 2 \mathfrak{s} L=2 \mathcal{O}$ then $\mathfrak{w} L=b \mathcal{O}$ and ord $a_{1}+$ ord $b$ is odd. Also ord $a_{1}=\operatorname{ord} \mathfrak{n} L \leq \operatorname{ord} 2 \mathfrak{s} L=e$ and ord $b=\operatorname{ord} \mathfrak{w} L<\operatorname{ord} 2 \mathfrak{s} L=e$. It follows that ord $a_{1} b<2 e$ and it is odd. Hence $d\left(-a_{1} a_{2}\right)=d\left(1-a_{1} b\right)=\operatorname{ord} a_{1} b=R_{1}+\operatorname{ord} b$ so $R_{2}-R_{1}+d\left(-a_{1} a_{2}\right)=-2 R_{1}+d\left(-a_{1} a_{2}\right)=-R_{1}+$ ord $b$. Also $\left(R_{2}-R_{1}\right) / 2+e=$ $-R_{1}+e>-R_{1}+\operatorname{ord} b$. It follows that $\alpha_{1}=-R_{1}+\operatorname{ord} b=-R_{1}+\operatorname{ord} \mathfrak{w} L$. So ord $\mathfrak{w} L=R_{1}+\alpha_{1}$.

We now prove the induction step. We have $L=L^{1} \perp L^{\prime}$, where $L^{\prime}=L^{2} \perp \ldots \perp$ $L^{m}$. Now let a and $\mathrm{a}^{\prime}$ be norm generators for $L^{1}$ and $L^{\prime}$. We have $\mathfrak{n} L^{1}=\mathfrak{n} L$ so a is also a norm generator for $L$. By Lemma 2.11 we have $\mathfrak{w} L=\mathfrak{w} L^{1}+\mathfrak{w} L^{\prime}+\mathrm{a}^{-1} \mathfrak{d}\left(\right.$ aa $\left.^{\prime}\right)$. $\left(\mathrm{a}^{-1} \mathfrak{d}(\mathrm{aa})=0\right.$ and $2 \mathfrak{s} L=2 \mathfrak{s} L^{1} \subseteq \mathfrak{w} L^{1}$ can be ignored.) Since ord $\mathrm{a}^{-1} \mathfrak{d}\left(\mathrm{aa}^{\prime}\right)=$ ord $\mathrm{a}^{\prime}+d\left(\mathrm{aa}^{\prime}\right)$ it follows that ord $\mathfrak{w} L=\min \left\{\right.$ ord $\mathfrak{w} L^{1}$, ord $\mathfrak{w} L^{\prime}$, ord $\left.\mathrm{a}^{\prime}+d\left(\mathrm{aa}^{\prime}\right)\right\}$.

If $L^{1}$ is unary then $R_{1} \leq R_{2}, L^{1} \cong \prec a_{1} \succ$ and $L^{\prime} \cong \prec a_{2}, \ldots, a_{n} \succ$. We take $\mathrm{a}=a_{1}$ and $\mathrm{a}^{\prime}=-a_{2}$. We have ord $\mathrm{a}^{\prime}=R_{2}, \mathfrak{w} L^{1}=R_{1}+e$ and ord $\mathfrak{w} L^{\prime}=$ $\min \left\{R_{2}+\alpha_{1}\left(L^{\prime}\right), R_{2}+e\right\}$. It follows that ord $\mathfrak{w} L=\min \left\{R_{1}+e, R_{2}+\alpha_{1}\left(L^{\prime}\right), R_{2}+\right.$ $\left.e, R_{2}+d\left(-a_{1} a_{2}\right)\right\}$. Since $R_{2}+e \geq R_{1}+e$, it can be removed. By Corollary 2.5 (ii) we have $\alpha_{1}=\min \left\{\left(R_{2}-R_{1}\right) / 2+e, R_{2}-R_{1}+d\left(-a_{1} a_{2}\right), R_{2}-R_{1}+\alpha_{1}\left(L^{\prime}\right)\right\}$. It follows that $\min \left\{R_{1}+\alpha_{1}, R_{1}+e\right\}=\min \left\{\left(R_{1}+R_{2}\right) / 2+e, R_{2}+d\left(-a_{1} a_{2}\right), R_{2}+\alpha_{1}\left(L^{\prime}\right), R_{1}+e\right\}$. But $R_{2} \geq R_{1}$, so $\left(R_{1}+R_{2}\right) / 2+e \geq R_{1}+e$. Thus $\min \left\{R_{1}+\alpha_{1}, R_{1}+e\right\}=$ $\min \left\{R_{2}+d\left(-a_{1} a_{2}\right), R_{2}+\alpha_{1}\left(L^{\prime}\right), R_{1}+e\right\}=\operatorname{ord} \mathfrak{w} L$.

If $L^{1}$ is binary then $R_{1} \geq R_{2}, L^{1} \cong \prec a_{1}, a_{2} \succ$ and $L^{\prime} \cong \prec a_{3}, \ldots, a_{n} \succ$. We prove that ord $\mathfrak{w} L=R_{1}+\alpha_{1}$. We take $\mathbf{a}=\pi^{2 u_{1}-2 r_{1}} a_{2}$ and $\mathfrak{a}^{\prime}=-a_{3}$. (See Lemma 2.13(iii).) We have $\mathfrak{w} L^{1}=R_{1}+\alpha_{1}\left(L^{1}\right), \mathfrak{w} L^{\prime}=\min \left\{R_{3}+\alpha_{1}\left(L^{\prime}\right), R_{3}+e\right\}$ and ord $\mathrm{a}^{\prime}+d\left(\mathrm{aa}^{\prime}\right)=R_{3}+d\left(-a_{2} a_{3}\right)$. Thus ord $\mathfrak{w} L=\min \left\{R_{1}+\alpha_{1}\left(L^{1}\right), R_{3}+\alpha_{1}\left(L^{\prime}\right), R_{3}+\right.$ $\left.d\left(-a_{2} a_{3}\right), R_{3}+e\right\}$. But $e \geq\left(R_{2}-R_{1}\right) / 2+e \geq \alpha_{1}\left(L^{\prime}\right)$, so $R_{3}+e \geq R_{3}+\alpha_{1}\left(L^{1}\right)$ and so $R_{3}+e$ can be removed. On the other hand $\alpha_{1}=\min \left\{\alpha_{1}\left(L^{1}\right), R_{3}-R_{1}+\right.$ $\left.d\left(-a_{2} a_{3}\right), R_{3}-R_{1}+\alpha_{1}\left(L^{\prime}\right)\right\}$. (We have $\alpha_{1}\left(L^{1}\right)=\min \left\{\left(R_{2}-R_{1}\right) / 2+e, R_{2}-R_{1}+\right.$ $\left.d\left(-a_{1} a_{2}\right)\right\}$ and, by Lemma 2.4(ii), $R_{3}-R_{1}+\alpha_{1}\left(L^{\prime}\right)=R_{3}-R_{1}+\alpha_{1}\left(\prec a_{3}, \ldots, a_{n} \succ\right)$ can replace all $R_{j+1}-R_{1}+d\left(-a_{j} a_{j+1}\right)$ with $j \geq 3$.) So $R_{1}+\alpha_{1}=\min \left\{R_{1}+\right.$ $\left.\alpha_{1}\left(L^{1}\right), R_{3}+d\left(-a_{2} a_{3}\right), R_{3}+\alpha_{1}\left(L^{\prime}\right)\right\}=$ ord $\mathfrak{w} L$.

Lemma 2.15. If $L_{k}$ is unary then $\mathfrak{w}_{k}=\mathfrak{s}_{k}\left(\mathfrak{f}_{k-1}+\mathfrak{f}_{k}+2 \mathcal{O}\right)$. (The term $\mathfrak{f}_{k-1}$ is ignored if $k=1$ and $\mathfrak{f}_{k}$ is ignored if $k=t$.)

Proof. Since $L_{k}$ is unary we have $\mathfrak{s}_{k}=\mathrm{a}_{k} \mathcal{O}$ and $u_{k}=r_{k}$. Also $\mathfrak{w} L_{k}=2 \mathfrak{s}_{k}$.

We have $L^{\mathfrak{s}_{k}}=\left(\perp_{j<k} \mathfrak{s}_{k} \mathfrak{s}_{j}^{-1} L_{j}\right) \perp L_{k} \perp\left(\perp_{j>k} L_{j}\right)$. The first orthogonal sum is included in $\mathfrak{s}_{k-1}^{-1} \mathfrak{s}_{k} L^{\mathfrak{s}_{k-1}}$, while the last one is included in $L^{\mathfrak{s}_{k+1}}$. Hence $L^{\mathfrak{s}_{k}} \subseteq L_{k}+\mathfrak{s}_{k-1}^{-1} \mathfrak{s}_{k} L^{\mathfrak{s}_{k-1}}+L^{\mathfrak{s}_{k+1}}$. The reverse inclusion follows from [4, 93:24] so $L^{\mathfrak{s}_{k}}=L_{k}+\mathfrak{s}_{k-1}^{-1} \mathfrak{s}_{k} L^{\mathfrak{s}_{k-1}}+L^{\mathfrak{s}_{k+1}}$. Now $\mathrm{a}_{k}$ is a norm generator for both $L^{\mathfrak{s}_{k}}$ and $L_{k}, \pi^{2\left(r_{k}-r_{k-1}\right)} \mathrm{a}_{k-1}$ for $\mathfrak{s}_{k-1}^{-1} \mathfrak{s}_{k} L^{\mathfrak{s}_{k-1}}$ and $\mathbf{a}_{k+1}$ for $L^{\mathfrak{s}_{k+1}}$. By Lemma 2.11 we have $\mathfrak{w}_{k}=\mathrm{a}_{k}^{-1} \mathfrak{d}\left(\pi^{2\left(r_{k}-r_{k-1}\right)} \mathrm{a}_{k-1} \mathrm{a}_{k}\right)+\mathrm{a}_{k}^{-1} \mathfrak{d}\left(\mathrm{a}_{k} \mathrm{a}_{k+1}\right)+\mathfrak{w} L_{k}+\mathfrak{w}\left(\mathfrak{s}_{k-1}^{-1} \mathfrak{s}_{k} L^{\mathfrak{s}_{k-1}}\right)+\mathfrak{w} L^{\mathfrak{s}_{k+1}}+$ 
$2 \mathfrak{s}_{k}=\mathfrak{s}_{k-1}^{-2} \mathfrak{s}_{k} \mathfrak{d}\left(\mathrm{a}_{k-1} \mathrm{a}_{k}\right)+\mathfrak{s}_{k}^{-1} \mathfrak{d}\left(\mathrm{a}_{k} \mathrm{a}_{k+1}\right)+\mathfrak{s}_{k-1}^{-2} \mathfrak{s}_{k}^{2} \mathfrak{w}_{k-1}+\mathfrak{w}_{k+1}+2 \mathfrak{s}_{k}$. (We ignore $\left.\mathrm{a}_{k} \mathfrak{d}\left(\mathrm{a}_{k} \mathrm{a}_{k}\right)=0.\right)$

If $u_{k}+u_{k+1}$ is even then by [4, 93:26] we have $\mathfrak{s}_{k}^{2} \mathfrak{f}_{k}=\mathfrak{d}\left(\mathrm{a}_{k} \mathrm{a}_{k+1}\right)+\mathrm{a}_{k+1} \mathfrak{w}_{k}+$ $\left.\mathrm{a}_{k} \mathfrak{w}_{k+1}+2 \mathfrak{p}^{\left(u_{k}+u_{k+1}\right) / 2+r_{k}}\right)$. This formula also holds in the case when $u_{k}+u_{k+1}$ is odd if we drop the last term. Indeed, in this case $\mathfrak{s}_{k}^{2} \mathfrak{f}_{k}=\mathrm{a}_{k} \mathrm{a}_{k+1} \mathcal{O}$ but ord $\mathrm{a}_{k} \mathrm{a}_{k+1}$ is odd so $\mathfrak{d}\left(\mathrm{a}_{k} \mathrm{a}_{k+1}\right)=\mathrm{a}_{k} \mathrm{a}_{k+1} \mathcal{O}$ and we also have $\mathrm{a}_{k+1} \mathfrak{w}_{k}, \mathrm{a}_{k} \mathfrak{w}_{k+1} \subseteq \mathrm{a}_{k} \mathrm{a}_{k+1} \mathcal{O}$. It follows that $\mathfrak{s}_{k}\left(\mathfrak{f}_{k-1}+\mathfrak{f}_{k}+2 \mathcal{O}\right)=\mathfrak{s}_{k}\left(\mathfrak{s}_{k-1}^{-2} \mathfrak{d}\left(\mathrm{a}_{k-1} \mathrm{a}_{k}\right)+\mathfrak{s}_{k-1}^{-2} \mathrm{a}_{k} \mathfrak{w}_{k-1}+\mathfrak{s}_{k-1}^{-2} \mathrm{a}_{k-1} \mathfrak{w}_{k}+\right.$ $2 \mathfrak{p}^{\left(u_{k-1}+u_{k}\right) / 2-r_{k-1}}+\mathfrak{s}_{k}^{-2} \mathfrak{d}\left(\mathrm{a}_{k} \mathrm{a}_{k+1}\right)+\mathfrak{s}_{k}^{-2} \mathrm{a}_{k+1} \mathfrak{w}_{k}+\mathfrak{s}_{k}^{-2} \mathrm{a}_{k} \mathfrak{w}_{k+1}+2 \mathfrak{p}^{\left(u_{k}+u_{k+1}\right) / 2-r_{k}}+$ 2O). (If $u_{k}+u_{k+1}$ is odd we ignore $2 \mathfrak{p}^{\left(u_{k}+u_{k+1}\right) / 2-r_{k}}$. If $u_{k-1}+u_{k}$ is odd we ignore $2 \mathfrak{p}^{\left(u_{k-1}+u_{k}\right) / 2-r_{k-1}}$.) But $r_{k}=u_{k}$, so $\left(u_{k-1}+u_{k}\right) / 2-r_{k-1}=\left(u_{k-1}-\right.$ $\left.2 r_{k-1}+2 r_{k}-u_{k}\right) / 2 \geq 0$ and $\left(u_{k}+u_{k+1}\right) / 2-r_{k}=\left(u_{k+1}-u_{k}\right) / 2 \geq 0$. Hence $2 \mathfrak{p}^{\left(u_{k-1}+u_{k}\right) / 2-r_{k-1}}, 2 \mathfrak{p}^{\left(u_{k}+u_{k+1}\right) / 2-r_{k}} \subseteq 2 \mathcal{O}$ so these terms can be ignored. Thus $\mathfrak{s}_{k}\left(\mathfrak{f}_{k-1}+\mathfrak{f}_{k}+2 \mathcal{O}\right)=\mathfrak{s}_{k-1}^{-2} \mathfrak{s}_{k} \mathfrak{d}\left(\mathrm{a}_{k-1} \mathrm{a}_{k}\right)+\mathfrak{s}_{k-1}^{-2} \mathfrak{s}_{k}^{2} \mathfrak{w}_{k-1}+\mathfrak{s}_{k-1}^{-2} \mathfrak{s}_{k} \mathrm{a}_{k-1} \mathfrak{w}_{k}+\mathfrak{s}_{k}^{-1} \mathfrak{d}\left(\mathrm{a}_{k} \mathrm{a}_{k+1}\right)+$ $\mathfrak{s}_{k}^{-1} \mathrm{a}_{k+1} \mathfrak{w}_{k}+\mathfrak{w}_{k+1}+2 \mathfrak{s}_{k}=\mathfrak{w}_{k}+\mathfrak{s}_{k-1}^{-2} \mathfrak{s}_{k} \mathrm{a}_{k-1} \mathfrak{w}_{k}+\mathfrak{s}_{k}^{-1} \mathrm{a}_{k+1} \mathfrak{w}_{k}=\mathfrak{w}_{k}$. (We have $\mathfrak{s}_{k-1}^{-2} \mathfrak{s}_{k} \mathrm{a}_{k-1}=\mathfrak{s}_{k-1}^{-2} \mathrm{a}_{k-1}\left(\mathfrak{s}_{k}^{-2} \mathrm{a}_{k}\right)^{-1} \subseteq \mathcal{O}$ and $\mathfrak{s}_{k}^{-1} \mathrm{a}_{k+1}=\mathrm{a}_{k}^{-1} \mathrm{a}_{k+1} \mathcal{O} \subseteq \mathcal{O}$.)

Lemma 2.16. Let $1 \leq i \leq n-1$. Then:

(i) If $n_{k-1}<i<n_{k}$ for some $1 \leq k \leq t$, then $R_{i}+\alpha_{i}=$ ord $\mathfrak{w}_{k}$ and $-R_{i+1}+\alpha_{i}=$ ord $\mathfrak{w}_{t-k}^{\sharp}$.

(ii) Suppose that $i=n_{k}$ for some $1 \leq k \leq t-1$. If $R_{i+1}-R_{i}$ is even or $\leq 2 e$ then $\alpha_{i}=$ ord $\mathfrak{f}_{k}$; otherwise $\alpha_{i}=\left(R_{i+1}-R_{i}\right) / 2+e$, ord $\mathfrak{f}_{k}=R_{i+1}-R_{i}=2 \alpha_{i}-2 e$ and both $\alpha_{i}$ and ord $\mathfrak{f}_{k}$ are $>2 e$.

Proof. (i) Note that $R_{i}+R_{i+1}=u_{k}+2 r_{k}-u_{k}=2 r_{k}$. Thus if $R_{i}+\alpha_{i}=$ ord $\mathfrak{w}_{k}$ then $-R_{i+1}+\alpha_{i}=$ ord $\mathfrak{w}_{k}-2 r_{k}=$ ord $\mathfrak{s}_{k}^{-2} \mathfrak{w}_{k}=$ ord $\mathfrak{w}_{t-k}^{\sharp}$ so it is enough to prove the first part of the statement. Also $R_{n_{k-1}+1}+R_{n_{k-1}+2}=R_{n_{k}-1}+R_{n_{k}}=2 r_{k}$ and so $R_{n_{k-1}+1}+\alpha_{n_{k-1}+1}=\ldots=R_{n_{k}-1}+\alpha_{n_{k}-1}$ by Corollary 2.3(i). Thus it is enough to prove our statement for only one value of $n_{k-1}<i<n_{k}$, say $i=n_{k-1}+1$.

We use induction on $t$. Note that if $k=1$ then ord $\mathfrak{w}_{1}=$ ord $\mathfrak{w} L=R_{1}+\alpha_{1}$ by Lemma 2.14 so we are done. In particular, (i) is true when $t=1$. Suppose now that $t \geq 2$. We may assume that $k \geq 2$. We have $L^{\mathfrak{s}_{k}}=\mathfrak{s}_{k} L_{(k-1)}^{\sharp} \perp L_{(k)}^{*}$. Since $i=n_{k-1}+1<n_{k}$ we have $R_{i-1}=2 r_{k-1}-u_{k-1}, R_{i}=u_{k}, R_{i+1}=2 r_{k}-u_{k}$, $L_{(k-1)} \cong \prec a_{1}, \ldots, a_{i-1} \succ$ and $L_{(k)}^{*} \cong \prec a_{i}, \ldots, a_{n} \succ$. Note that $R_{i} \geq R_{i+1}$. If a and $\mathrm{b}$ are norm generators for $L_{(k)}^{*}$ and $\mathfrak{s}_{k} L_{(k-1)}^{\sharp}$, respectively, then $\mathfrak{n} L_{(k)}^{*}=\mathfrak{p}^{R_{i}}=$ $\mathfrak{p}^{u_{k}}=\mathfrak{n} L^{\mathfrak{s}_{k}}$. Therefore a is also a norm generator for $L^{\mathfrak{s}_{k}}$. By Lemma 2.11 we have $\mathfrak{w}_{k}=\mathfrak{w} L^{\mathfrak{s}_{k}}=\mathfrak{w}\left(\mathfrak{s}_{k} L_{(k-1)}^{\sharp}\right)+\mathfrak{w} L_{(k)}^{*}+\mathrm{a}^{-1} \mathfrak{d}(\mathrm{ab})+2 \mathfrak{s}_{k}$, which implies that ord $\mathfrak{w}_{k}=$ $\min \left\{\operatorname{ord} \mathfrak{w}\left(\mathfrak{s}_{k} L_{(k-1)}^{\sharp}\right)\right.$, ord $\mathfrak{w} L_{(k)}^{*}$, ord $\left.\mathfrak{b}+d(\mathrm{ab}), r_{k}+e\right\}$. Now $L_{k}$ is not unary so $\operatorname{ord} \mathfrak{w} L_{(k)}^{*}=R_{i}+\alpha_{1}\left(L_{(k)}^{*}\right)$ by Lemma 2.14. Also $L_{(k-1)}^{\sharp} \cong \prec a_{i-1}^{-1}, \ldots, a_{1}^{-1} \succ$ and $\operatorname{ord} a_{i-1}^{-1}=-R_{i-1}$ so by Lemma 2.14 we have ord $\mathfrak{w} L_{(k-1)}^{\sharp}=\min \left\{-R_{i-1}+\right.$ $\left.\alpha_{1}\left(L_{(k-1)}^{\sharp}\right),-R_{i-1}+e\right\}=\min \left\{-R_{i-1}+\alpha_{i-2}\left(L_{(k-1)}\right),-R_{i-1}+e\right\}$. (We have $\alpha_{1}\left(L_{(k-1)}^{\sharp}\right)=\alpha_{i-2}\left(L_{(k-1)}\right)$ by 2.6.) It follows that ord $\mathfrak{w}\left(\mathfrak{s}_{k} L_{(k-1)}^{\sharp}\right)=\min \left\{2 r_{k}-\right.$ $\left.R_{i-1}+\alpha_{i-2}\left(L_{(k-1)}\right), 2 r_{k}-R_{i-1}+e\right\}=\min \left\{R_{i}+R_{i+1}-R_{i-1}+\alpha_{i-2}\left(L_{(k-1)}\right), R_{i}+\right.$ $\left.R_{i+1}-R_{i-1}+e\right\}$. Now $a_{i-1}^{-1}$ is a norm generator for $L_{(k-1)}^{\sharp}$, so b := $\pi^{2 r_{k}} a_{i-1}^{-1}$ is a norm generator for $\mathfrak{s}_{k} L_{(k-1)}^{\sharp}$, and $a:=-a_{i}$ is a norm generator for $L_{(k)}$. We get ord $\mathrm{b}+d(\mathrm{ab})=2 r_{k}-R_{i-1}+d\left(-\pi^{2 r_{k}} a_{i-1}^{-1} a_{i}\right)=R_{i}+R_{i+1}-R_{i-1}+d\left(-a_{i-1} a_{i}\right)$. Also 
$r_{k}+e=\left(R_{i}+R_{i+1}\right) / 2+e$. Thus ord $\mathfrak{w}_{k}=\min \left\{R_{i}+\alpha_{1}\left(L_{(k)}^{*}\right), R_{i}+R_{i+1}-R_{i-1}+\right.$ $\left.d\left(-a_{j-1} a_{j}\right), R_{i}+R_{i+1}-R_{i-1}+\alpha_{i-2}\left(L_{(k-1)}\right), R_{i}+R_{i+1}-R_{i-1}+e,\left(R_{i}+R_{i+1}\right) / 2+e\right\}$. But $R_{i-1} \leq R_{i+1} \leq R_{i}$, so $R_{i}+R_{i+1}-R_{i-1}+e \geq\left(R_{i}+R_{i+1}\right) / 2+e=$ $R_{i}+\left(R_{i+1}-R_{i}\right) / 2+e \geq R_{i}+\alpha_{1}\left(L_{(k)}^{*}\right)$, so the last two terms can be removed. By Lemma 2.4(i), $R_{i+1}-R_{i-1}+\alpha_{i-2}\left(L_{(k-1)}\right)=R_{i+1}-R_{i-1}+\alpha_{i-2}\left(\prec a_{1}, \ldots, a_{i-1} \succ\right)$ replaces all the terms in the definition of $\alpha_{i}$ with $1 \leq j \leq i-2$, while by Lemma 2.1, $\alpha_{1}\left(L_{(k)}^{*}\right)=\alpha_{1}\left(\prec a_{i}, \ldots, a_{n} \succ\right)$ replaces $\left(R_{i+1}-R_{i}\right) / 2+e$ and the terms with $i \leq j<$ $n$. Hence $\alpha_{i}=\min \left\{R_{i+1}-R_{i-1}+\alpha_{i-2}\left(L_{(k-1)}\right), R_{i+1}-R_{i-1}+d\left(-a_{i-1} a_{i}\right), \alpha_{1}\left(L_{(k)}^{*}\right)\right\}$. It follows that $R_{i}+\alpha_{i}=\min \left\{R_{i}+R_{i+1}-R_{i-1}+\alpha_{i-2}\left(L_{(k-1)}\right), R_{i}+R_{i+1}-R_{i-1}+\right.$ $\left.d\left(-a_{i-1} a_{i}\right), R_{i}+\alpha_{1}\left(L_{(k)}^{*}\right)\right\}=$ ord $\mathfrak{w}_{k}$.

(ii) Since $i=n_{k}$ we have $R_{i}=2 r_{k}-u_{k}, R_{i+1}=u_{k+1}, L_{(k)} \cong \prec a_{1}, \ldots, a_{i} \succ$ and $L_{(k+1)}^{*} \cong \prec a_{i+1}, \ldots, a_{n} \succ$. We have $R_{i+1}-R_{i}=u_{k}+u_{k+1}-2 r_{k}$, so $R_{i+1}-R_{i}$ is even iff $u_{k}+u_{k+1}$ is even. If $u_{k}+u_{k+1}$ and $R_{i+1}-R_{i}$ are odd then $\mathfrak{f}_{k}=\mathfrak{s}_{k}^{-2} \mathrm{a}_{k} \mathrm{a}_{k+1}$ so ord $\mathfrak{f}_{k}=u_{k}+u_{k+1}-2 r_{k}=R_{i+1}-R_{i}$. If $R_{i+1}-R_{i}<2 e$ then $\alpha_{i}=R_{i+1}-R_{i}=$ ord $\mathfrak{f}_{k}$, while if $R_{i+1}-R_{i}>2 e$ then $\alpha_{i}=\left(R_{i+1}-R_{i}\right) / 2+e$. (See Lemma 2.7(ii) and (iii).)

Suppose now that $R_{i+1}-R_{i}$ is even. By Lemma 2.12 we have $\mathfrak{f}_{k}=\mathfrak{s}_{k}^{-2} \mathfrak{d}\left(\mathrm{a}_{k} \mathrm{a}_{k+1}\right)+$ $\mathrm{a}_{k} \mathfrak{s}_{k}^{-2} \mathfrak{w} L_{(k+1)}^{*}+\mathrm{a}_{k+1} \mathfrak{w} L_{(k)}^{\sharp}+2 \mathfrak{p}^{\left(u_{k}+u_{k+1}\right) / 2-r_{k}}$. We take $\mathrm{a}_{k}=\pi^{2 u_{k}-2 r_{k}} a_{i}$ and $\mathrm{a}_{k+1}=-a_{i+1}$. (See Lemma 2.13(iii).) Thus ord $\mathfrak{d}\left(\mathrm{a}_{k} \mathrm{a}_{k+1}\right)=\operatorname{ord}\left(\mathrm{a}_{k} \mathrm{a}_{k+1}\right)+$ $d\left(\mathrm{a}_{k} \mathrm{a}_{k+1}\right)=u_{k}+u_{k+1}+d\left(-a_{i} a_{i+1}\right)$ and so ord $\mathfrak{s}_{k}^{-2} \mathfrak{d}\left(\mathrm{a}_{k} \mathrm{a}_{k+1}\right)=-2 r_{k}+u_{k}+u_{k+1}+$ $d\left(-a_{i} a_{i+1}\right)=R_{i+1}-R_{i}+d\left(-a_{i} a_{i+1}\right)$. By Lemma 2.14 we have ord $\mathfrak{w} L_{(k+1)}^{*}=$ $\min \left\{R_{i+1}+\alpha_{1}\left(L_{k+1}^{*}\right), R_{i+1}+e\right\}$. Since ord $a_{k} \mathfrak{s}_{k}^{-2}=u_{k}-2 r_{k}=-R_{i}$, we get $\operatorname{ord}\left(\mathrm{a}_{k} \mathfrak{s}_{k}^{-2} \mathfrak{w} L_{(k+1)}^{*}\right)=\min \left\{R_{i+1}-R_{i}+\alpha_{1}\left(L_{(k+1)}^{*}\right), R_{i+1}-R_{i}+e\right\}$. We have $L_{(k)}^{\sharp} \cong$ $\prec a_{i}^{-1}, \ldots, a_{1}^{-1} \succ$, so ord $\mathfrak{w} L_{(k)}^{\sharp}=\min \left\{-R_{i}+\alpha_{1}\left(L_{(k)}^{\sharp}\right),-R_{i}+e\right\}$. Since $\alpha_{1}\left(L_{(k)}^{\sharp}\right)=$ $\alpha_{i-1}\left(L_{(k)}\right)$ (see Remark 2.6) and ord $\mathrm{a}_{k+1}=u_{k+1}=R_{i+1}$ we have ord $\left(\mathrm{a}_{k+1} \mathfrak{w} L_{(k)}^{\sharp}\right)=$ $\min \left\{R_{i+1}-R_{i}+\alpha_{i-1}\left(L_{(k)}\right), R_{i+1}-R_{i}+e\right\}$. Finally ord $2 \mathfrak{p}^{\left(u_{k}+u_{k+1}\right) / 2-r_{k}}=\left(u_{k}+\right.$ $\left.u_{k+1}\right) / 2-r_{k}+e=\left(R_{i+1}-R_{i}\right) / 2+e$. Thus ord $\mathfrak{f}_{k}=\min \left\{\left(R_{i+1}-R_{i}\right) / 2+e, R_{i+1}-\right.$ $\left.R_{i}+d\left(-a_{i} a_{i+1}\right), R_{i+1}-R_{i}+\alpha_{i-1}\left(L_{(k)}\right), R_{i+1}-R_{i}+\alpha_{1}\left(L_{(k+1)}^{*}\right), R_{i+1}-R_{i}+e\right\}$. But $R_{i}=2 r_{k}-u_{k} \leq u_{k} \leq u_{k+1}=R_{i+1}$ so $R_{i+1}-R_{i}+e \geq\left(R_{i+1}-R_{i}\right) / 2+e$, so it can be ignored. So ord $\mathfrak{f}_{k}=\min \left\{\left(R_{i+1}-R_{i}\right) / 2+e, R_{i+1}-R_{i}+d\left(-a_{i} a_{i+1}\right), R_{i+1}-\right.$ $\left.R_{i}+\alpha_{i-1}\left(L_{(k)}\right), R_{i+1}-R_{i}+\alpha_{1}\left(L_{(k+1)}^{*}\right)\right\}$, which, by Corollary 2.5 (ii), is equal to $\alpha_{i}$. (Recall, $L_{(k)} \cong \prec a_{1}, \ldots, a_{i} \succ$ and $L_{(k+1)}^{*} \cong \prec a_{i+1}, \ldots, a_{n} \succ$.)

Corollary 2.17. (i) If $L_{k}$ is not unary and $i=n_{k-1}+1$ or $n_{k}-1$ then ord $a_{k}^{-1} \mathfrak{w}_{k}=$ $\alpha_{i}$.

(ii) If $L_{k}$ is unary and $i=n_{k}$ then $\operatorname{ord~}_{k}^{-1} \mathfrak{w}_{k}=\min \left\{\alpha_{i-1}, \alpha_{i}, e\right\}$. (We ignore $\alpha_{i-1}$ if $i=1$, and $\alpha_{i}$ if $i=n$.)

Proof. (i) In both cases when $i=n_{k-1}+1$ or $n_{k}-1$ we have $R_{i}=u_{k}=$ ord $\mathrm{a}_{k}$. Hence ord $\mathfrak{w}_{k}=R_{i}+\alpha_{i}=$ ord $\mathrm{a}_{k}+\alpha_{i}$ so ord $\mathrm{a}_{k}^{-1} \mathfrak{w}_{k}=\alpha_{i}$.

(ii) We have $\mathfrak{s}_{k}=\mathrm{a}_{k} \mathcal{O}$ and, by Lemma $2.15, \mathfrak{w}_{k}=\mathfrak{s}_{k}\left(\mathfrak{f}_{k-1}+\mathfrak{f}_{k}+2 \mathcal{O}\right)$ so $a_{k}^{-1} \mathfrak{w}_{k}=$ $\mathfrak{f}_{k-1}+\mathfrak{f}_{k}+2 \mathcal{O}$. Thus ord $a_{k}^{-1} \mathfrak{w}_{k}=\min \left\{\right.$ ord $\mathfrak{f}_{k-1}$, ord $\left.\mathfrak{f}_{k}, e\right\}$ and we have to prove that it is equal to $\min \left\{\alpha_{i-1}, \alpha_{i}, e\right\}$. Now $i-1=n_{k}-1=n_{k-1}$ so, by Lemma 2.16(ii), we have either $\alpha_{i-1}=$ ord $\mathfrak{f}_{k-1}$ or $\alpha_{i-1}$, ord $\mathfrak{f}_{k-1}>2 e$. But if $\alpha_{i-1}$, ord $\mathfrak{f}_{k-1}>$ $2 e>e$ then they can be ignored in $\min \left\{\alpha_{i-1}, \alpha_{i}, e\right\}$ and $\min \left\{\right.$ ord $\mathfrak{f}_{k-1}$, ord $\left.\mathfrak{f}_{k}, e\right\}$, respectively. Similarly either $\alpha_{i}=\operatorname{ord} \mathfrak{f}_{k}$ or $\alpha_{i}$, ord $\mathfrak{f}_{k}$ are both $>2 e>e$ so they can be ignored. Thus $\min \left\{\alpha_{i-1}, \alpha_{i}, e\right\}=\min \left\{\operatorname{ord} \mathfrak{f}_{k-1}, \operatorname{ord} \mathfrak{f}_{k}, e\right\}$. 


\section{MAIN THEOREM}

In this section we state and prove the main result of this paper, the classification of integral lattices over dyadic local fields in terms of good BONGs. It is well known that this problem was first solved by O'Meara in [4, Theorem 93:28]. Since our proof uses O'Meara's result we first state Theorem 93:28.

Throughout this section $L, K$ are two lattices with $L \cong \prec a_{1}, \ldots, a_{n} \succ$ and $K \cong \prec b_{1}, \ldots, b_{n} \succ$ relative to good BONGs. In terms of Jordan decompositions we write $L=L_{1} \perp \ldots \perp L_{t}$ and $K=K_{1} \perp \ldots \perp K_{t^{\prime}}$. Let $\mathfrak{s}_{k}=\mathfrak{s} L_{k}, \mathfrak{s}_{k}^{\prime}=\mathfrak{s} K_{k}$, $\mathfrak{g}_{k}=\mathfrak{g} L^{\mathfrak{s} k}, \mathfrak{g}_{k}^{\prime}=\mathfrak{g} K^{\mathfrak{s}_{k}^{\prime}}, \mathfrak{w}_{k}=\mathfrak{w} L^{\mathfrak{s}_{k}}, \mathfrak{w}_{k}^{\prime}=\mathfrak{w} K^{\mathfrak{s}_{k}^{\prime}}, \mathfrak{f}_{k}=\mathfrak{f}_{k}(L)$ and $\mathfrak{f}_{k}^{\prime}=\mathfrak{f}_{k}(K)$. Let $\mathrm{a}_{k}$ and $\mathrm{b}_{k}$ be norm generators for $L^{\mathfrak{s}_{k}}$ and $K^{\mathfrak{s}_{k}^{\prime}}$, respectively. We say that $L$ and $K$ are of the same fundamental type if

$$
t=t^{\prime}, \operatorname{dim} L_{k}=\operatorname{dim} K_{k}, \mathfrak{s}_{k}=\mathfrak{s}_{k}^{\prime}, \mathfrak{g}_{k}=\mathfrak{g}_{k}^{\prime}
$$

for $1 \leq k \leq t$. These conditions are equivalent to

$$
t=t^{\prime}, \operatorname{dim} L_{k}=\operatorname{dim} K_{k}, \mathfrak{s}_{k}=\mathfrak{s}_{k}^{\prime}, \mathfrak{w}_{k}=\mathfrak{w}_{k}^{\prime}, \mathrm{a}_{k} \cong \mathrm{b}_{k} \quad\left(\bmod \mathfrak{w}_{k}\right)
$$

for $1 \leq k \leq t$. We now state O'Meara's Theorem 93:28.

Theorem 93:28. Let $L, K$ be lattices with the same fundamental type such that $F L \cong F K$. Let $L_{(1)} \subset \cdots \subset L_{(t)}$ and $K_{(1)} \subset \cdots \subset K_{(t)}$ be Jordan chains for $L$ and $K$. Then $L \cong K$ if and only if the following conditions hold for $1 \leq i \leq t-1$ :

(i) $\operatorname{det} L_{(k)} / \operatorname{det} K_{(k)} \cong 1\left(\bmod \mathfrak{f}_{k}\right)$;

(ii) $F L_{(k)} \rightarrow F K_{(k)} \perp\left[\mathrm{a}_{k+1}\right]$ when $\mathfrak{f}_{k} \subset 4 \mathrm{a}_{k+1} \mathfrak{w}_{k+1}^{-1}$;

(iii) $F L_{(k)} \rightarrow F K_{(k)} \perp\left[\mathrm{a}_{k}\right]$ when $\mathfrak{f}_{k} \subset 4 \mathrm{a}_{k} \mathfrak{w}_{k}^{-1}$.

We now state our main result.

Theorem 3.1. Let $L, K$ be two lattices with $F L \cong F K$ and let $L \cong \prec a_{1}, \ldots, a_{n} \succ$ and $K \cong \prec b_{1}, \ldots, b_{n} \succ$ relative to good BONGs. Let $R_{i}=R_{i}(L)=\operatorname{ord} a_{i}, S_{i}=$ $R_{i}(K)=\operatorname{ord} b_{i}, \alpha_{i}=\alpha_{i}(L)$ and $\beta_{i}=\alpha_{i}(K)$. Then $L \cong K$ iff:

(i) $R_{i}=S_{i}$ for $1 \leq i \leq n$;

(ii) $\alpha_{i}=\beta_{i}$ for $1 \leq i \leq n-1$;

(iii) $d\left(a_{1} \cdots a_{i} b_{1} \cdots b_{i}\right) \geq \alpha_{i}$ for $1 \leq i \leq n-1$;

(iv) $\left[b_{1}, \ldots, b_{i-1}\right] \rightarrow\left[a_{1}, \ldots, a_{i}\right]$ for any $1<i<n$ s.t. $\alpha_{i-1}+\alpha_{i}>2 e$.

Proof. Condition 3.1(i) is equivalent to $t=t^{\prime}, \operatorname{dim} L_{k}=\operatorname{dim} K_{k}, \mathfrak{s}_{k}=\mathfrak{s}_{k}^{\prime}$ and $\mathfrak{n} L^{\mathfrak{s}_{k}}=\mathfrak{n} K^{\mathfrak{s}_{k}}$, i.e. $\mathrm{a}_{k} \mathcal{O}=\mathrm{b}_{k} \mathcal{O}$. (See [1, Lemma 4.7].) Suppose this happens. Denote as before $n_{k}=\operatorname{dim} L_{(k)}=\operatorname{dim} K_{(k)}, \mathfrak{p}^{r_{k}}=\mathfrak{s}_{k}$ and $\mathfrak{p}^{u_{k}}=\mathfrak{n} L^{\mathfrak{s}_{k}}=\mathrm{a}_{k} \mathcal{O}$.

As in the previous section, we choose a Jordan splitting of $L$ such that $L_{k} \cong$ $\prec a_{n_{k-1}+1}, \ldots, a_{n_{k}} \succ$. Hence for any $1 \leq k \leq n, a_{k}$ can be either $\pm a_{n_{k-1}+1}$ or $\pm \pi^{2 u_{k}-2 r_{k}} a_{n_{k}}$. We choose a Jordan splitting for $K$ with the same property.

Assuming that 3.1(i) holds, Lemma 2.16 and Corollary 2.17(ii) imply that 3.1(ii) is equivalent to $\mathfrak{w}_{k}=\mathfrak{w}_{k}^{\prime}$ for $1 \leq k \leq t$ and $\mathfrak{f}_{k}=\mathfrak{f}_{k}^{\prime}$ for $1 \leq k \leq t-1$.

From here the proof of Theorem 3.1 consists of two steps:

1. Assuming that 3.1(i) and (ii) hold, we prove that condition 3.1(iii) is equivalent to $\mathrm{a}_{k} \cong \mathrm{b}_{k}\left(\bmod \mathfrak{w}_{k}\right)$ for any $1 \leq k \leq t$ and condition 93:28(i).

2. Assuming that 3.1(i)-(iii) hold, we prove that condition 3.1(iv) is equivalent to conditions $93: 28$ (ii) and (iii). 
Lemma 3.2. Suppose that $L, K$ satisfy conditions 3.1(i) and 3.1(ii). If $R_{i-1}=$ $R_{i+1}$ for some $1<i<n$ then:

(i) If 3.1(iii) holds at $i-2$ or $i-2=0$ then 3.1(iii) holds at $i$.

(ii) If 3.1(iii) holds at $i+1$ or $i+1=n$ then 3.1(iii) holds at $i-1$.

Proof. (i) We have $d\left(a_{1} \cdots a_{i} b_{1} \cdots b_{i}\right) \geq \min \left\{d\left(a_{1} \cdots a_{i-2} b_{1} \cdots b_{i-2}\right), d\left(-a_{i-1} a_{i}\right)\right.$, $\left.d\left(-b_{i-1} b_{i}\right)\right\}$. (If $i-2=0$ we ignore $d\left(a_{1} \cdots a_{i-2} b_{1} \cdots b_{i-2}\right)$.) But $d\left(a_{1} \cdots a_{i-2} b_{1} \cdots b_{i-2}\right) \geq \alpha_{i-2} \geq R_{i-1}-R_{i+1}+\alpha_{i}=\alpha_{i}$. (We have $-R_{i-1}+\alpha_{i-2} \geq$ $-R_{i+1}+\alpha_{i}$.) Also $d\left(-a_{i-1} a_{i}\right)=R_{i+1}-R_{i-1}+d\left(-a_{i-1} a_{i}\right) \geq \alpha_{i}$. Similarly $d\left(-b_{i-1} b_{i}\right) \geq \alpha_{i}$. Hence $d\left(a_{1} \cdots a_{i} b_{1} \cdots b_{i}\right) \geq \alpha_{i}$.

(ii) is similar. This time $R_{i+1}+\alpha_{i+1} \geq R_{i-1}+\alpha_{i-1}$ so $d\left(a_{1} \cdots a_{i+1}, b_{1} \cdots b_{i+1}\right) \geq$ $\alpha_{i+1} \geq R_{i-1}-R_{i+1}+\alpha_{i-1}=\alpha_{i-1}$. (If $i+1=n$ then $d\left(a_{1} \cdots a_{n} b_{1} \cdots b_{n}\right)=$ $\infty>\alpha_{n-2}$.) Also $d\left(-a_{i} a_{i+1}\right)=R_{i+1}-R_{i-1}+d\left(-a_{i} a_{i+1}\right) \geq \alpha_{i-1}$ and similarly $d\left(-b_{i} b_{i+1}\right) \geq \alpha_{i-1}$.

Lemma 3.3. Assuming that 3.1(i) and (ii) hold, condition 3.1(iii) is equivalent to $\mathrm{a}_{k} \cong \mathrm{b}_{k}\left(\bmod \mathfrak{w}_{k}\right)$ for any $1 \leq k \leq t$ and condition 93:28(i).

Proof. We have $L_{(k)} \cong \prec a_{1}, \ldots, a_{n_{k}} \succ$ and $K_{(k)} \cong \prec b_{1}, \ldots, b_{n_{k}} \succ$. Hence $\operatorname{det} L_{(k)}$ $=a_{1} \cdots a_{n_{k}}$ and $\operatorname{det} K_{(k)}=b_{1} \cdots b_{n_{k}}$. Since the two determinants have the same order, $R_{1}+\cdots+R_{n_{k}}$, the condition $\operatorname{det} L_{(k)} / \operatorname{det} K_{(k)} \cong 1\left(\bmod \mathfrak{f}_{k}\right)$ is equivalent to $d\left(a_{1} \cdots a_{n_{k}} b_{1} \cdots b_{n_{k}}\right) \geq$ ord $\mathfrak{f}_{k}$. Let $i=n_{k}$. We claim that $d\left(a_{1} \cdots a_{i} b_{1} \cdots b_{i}\right) \geq$ ord $\mathfrak{f}_{k}$ is equivalent to $d\left(a_{1} \cdots a_{i} b_{1} \cdots b_{i}\right) \geq \alpha_{i}$. By Lemma 2.16(ii) we have either $\alpha_{i}=\operatorname{ord} \mathfrak{f}_{k}$ or $\alpha_{i}$, ord $\mathfrak{f}_{k}>2 e$. In the first case our claim is obvious and in the second both $d\left(a_{1} \cdots a_{i} b_{1} \cdots b_{i}\right) \geq$ ord $\mathfrak{f}_{k}$ and $d\left(a_{1} \cdots a_{i} b_{1} \cdots b_{i}\right) \geq \alpha_{i}$ are equivalent to $a_{1} \cdots a_{i} b_{1} \cdots b_{i} \in \dot{F}^{2}$.

Thus condition 3.1(iii) at indices $i=n_{k}$ with $1 \leq k \leq t-1$ is equivalent to 93:28(i). Assume these equivalent conditions hold. We want to prove that condition $\mathrm{a}_{k} \cong \mathrm{b}_{k}\left(\bmod \mathfrak{w}_{k}\right)$ at indices $1 \leq k \leq t$ such that $L_{k}$ is not unary is equivalent to condition 3.1(iii) at $i=n_{k-1}+1$, while if $L_{k}$ is unary then it holds unconditionally.

Note that $\mathrm{a}_{k} \cong \mathrm{b}_{k}\left(\bmod \mathfrak{w}_{k}\right)$ is equivalent to $\mathrm{b}_{k} / \mathrm{a}_{k} \cong 1\left(\bmod \mathrm{a}_{k}^{-1} \mathfrak{w}_{k}\right)$, i.e. to $d\left(\mathrm{a}_{k} \mathrm{~b}_{k}\right)=d\left(\mathrm{~b}_{k} / \mathrm{a}_{k}\right) \geq \operatorname{ord} \mathrm{a}_{k}^{-1} \mathfrak{w}_{k}$. We will take $\mathrm{a}_{k}=a_{n_{k-1}+1}=a_{i}$ and $\mathrm{b}_{k}=$ $b_{n_{k-1}+1}=b_{i}$. So our condition is equivalent to $d\left(a_{i} b_{i}\right) \geq \operatorname{ord~a}_{k}^{-1} \mathfrak{w}_{k}$, where $i=$ $n_{k-1}+1$.

If $L_{k}$ is unary then ord $a_{k}^{-1} \mathfrak{w}_{k}=\min \left\{\alpha_{i-1}, \alpha_{i}, e\right\}$ by Corollary 2.17(ii), where $i=n_{k-1}+1=n_{k}$. Since $i-1=n_{k-1}$ and $i=n_{k}$, condition 3.1(iii) is satisfied for both. Thus $d\left(a_{1} \cdots a_{i-1} b_{1} \cdots b_{i-1}\right) \geq \alpha_{i-1} \geq \operatorname{ord}_{k}^{-1} \mathfrak{w}_{k}$ and $d\left(a_{1} \cdots a_{i} b_{1} \cdots b_{i}\right) \geq$ $\alpha_{i} \geq \operatorname{ord~}_{k}^{-1} \mathfrak{w}_{k}$ so $d\left(a_{i} b_{i}\right) \geq \operatorname{ord} \mathrm{a}_{k}^{-1} \mathfrak{w}_{k}$. (If $k=1$ so $i=n_{0}+1=1$ we ignore $\alpha_{i-1}$ and we have $d\left(a_{1} b_{1}\right) \geq \alpha_{1} \geq \operatorname{ord} a_{1}^{-1} \mathfrak{w}_{1}$. If $k=t$ so $i=n_{t}=n$ we ignore $\alpha_{i}$ and, since $a_{1} \cdots a_{n}=\operatorname{det} F M=\operatorname{det} F N=b_{1} \cdots b_{n}$ in $\dot{F} / \dot{F}^{2}$, we get $d\left(a_{n} b_{n}\right)=$ $\left.d\left(a_{1} \cdots a_{n-1} b_{1} \cdots b_{n-1}\right) \geq \alpha_{n-1} \geq \operatorname{ord} a_{t}^{-1} \mathfrak{w}_{t}.\right)$ Thus condition $\mathrm{a}_{k} \cong \mathrm{b}_{k}\left(\bmod \mathfrak{w}_{k}\right)$ is superfluous when $L_{k}$ is unary.

Suppose now that $L_{k}$ is not unary and let $i=n_{k-1}+1$. By Corollary 2.17(i) we have ord $\mathrm{a}_{k}^{-1} \mathfrak{w}_{k}=\alpha_{i}$. We will prove that $d\left(a_{i} b_{i}\right) \geq \operatorname{ord} \mathrm{a}_{k}^{-1} \mathfrak{w}_{k}=\alpha_{i}$ is equivalent to the condition 3.1(iii) at $i$ i.e. to $d\left(a_{1} \cdots a_{i} b_{1} \cdots b_{i}\right) \geq \alpha_{i}$. If $k=1$ so $i=n_{0}+1=1$ this is obvious. If $k>1$ so $i>1$ note that $-R_{i}+\alpha_{i-1} \geq-R_{i+1}+\alpha_{i}$ and $R_{i}=u_{k} \geq$ $2 r_{k}-u_{k}=R_{i+1}$ so $\alpha_{i-1} \geq \alpha_{i}$. We have $i-1=n_{k-1}$ so $d\left(a_{1} \cdots a_{i-1} b_{1} \cdots b_{i-1}\right) \geq$ $\alpha_{i-1} \geq \alpha_{i}$ and so $d\left(a_{1} b_{i}\right) \geq \alpha_{i}$ is equivalent to $d\left(a_{1} \cdots a_{i} b_{1} \cdots b_{i}\right) \geq \alpha_{i}$ by the domination principle. 
To complete the proof we show that 3.1(iii) is true if it is true for $i=n_{k}$, where $1 \leq k \leq t-1$, and for $i=n_{k-1}+1$, where $1 \leq k \leq t$ and $L_{k}$ is not unary. To do this we use Lemma 3.2.

Let $1 \leq k \leq t$. For any $n_{k-1}+1<i<n_{k}$ we have $R_{i-1}=R_{i+1}$ (they are both $u_{k}$ or $2 r_{k}-u_{k}$ ) so by Lemma 3.2(i) if 3.1(iii) holds for $i-2$ or $i-2=0$ it will also hold for $i$. Thus, since 3.1(iii) is true for $n_{k-1}$ (or $n_{k-1}=0$ if $k=1$ ), it will also be true by induction for any $n_{k-1}+2 \leq i<n_{k}$ with $i \equiv n_{k-1}(\bmod 2)$. Similarly since 3.1 (iii) is true at $n_{k-1}+1$, it will also be true by induction for any $n_{k-1}+1 \leq i<n_{k}$ with $i \equiv n_{k-1}+1(\bmod 2)$. Hence 3.1(iii) holds for any $n_{k-1}<i<n_{k}$. Since 3.1(iii) also holds for any $i=n_{k}$ with $1 \leq k \leq t-1$ it will hold for all $1 \leq i \leq n-1$.

Lemma 3.4. If $1<i<n$ and $R_{i-1}=R_{i+1}$ then $\alpha_{i-1}+\alpha_{i} \leq 2 e$.

Proof. We have $\alpha_{i-1}+\alpha_{i} \leq\left(R_{i}-R_{i-1}\right) / 2+e+\left(R_{i+1}-R_{i}\right) / 2+e=\left(R_{i+1}-\right.$ $\left.R_{i-1}\right) / 2+2 e$ so if $R_{i-1}=R_{i+1}$ then $\alpha_{i-1}+\alpha_{i} \leq 2 e$.

Lemma 3.5. Let $V, W$ be two quadratic spaces over $F$. We have: H.

(i) If $\operatorname{dim} V-\operatorname{dim} W=1$ and $\mathrm{H}$ is a hyperbolic plane then $W \rightarrow V$ iff $V \rightarrow W \perp$

(ii) If $\operatorname{dim} V=\operatorname{dim} W$ and $a \in \dot{F}$ then $W \rightarrow V \perp[a]$ iff $V \rightarrow W \perp[a \operatorname{det} V \operatorname{det} W]$.

(iii) If $\operatorname{dim} V=\operatorname{dim} W, a, b \in \dot{F}$ and $(a b, \operatorname{det} V \operatorname{det} W)_{\mathfrak{p}}=1$ (in particular, if $d(a b)+d(\operatorname{det} V \operatorname{det} W)>2 e)$ then $W \rightarrow V \perp[a]$ iff $W \rightarrow V \perp[b]$.

Proof. This is a direct consequence of [4, 63:21]. For (iii) we also use the fact that if $x y=z t$ then $[x, y] \cong[z, t]$ iff $z \rightarrow[x, y]$, which in turn is equivalent to $(x z, y z)_{\mathfrak{p}}=1$.

Lemma 3.6. Suppose that $L, K$ satisfy the conditions 3.1(i)-(iii) (or, equivalently, they have the same fundamental type and they satisfy the condition 93:28(i)). Then:

(i) If $\mathfrak{f}_{k} \subset 4 \mathrm{a}_{k} \mathfrak{w}_{k}^{-1}$ and both $\mathrm{a}_{k}$ and $\mathrm{b}_{k}$ are norm generators for $L^{\mathfrak{s}_{k}}$ then $F L_{(k)} \rightarrow F K_{(k)} \perp\left[\mathrm{a}_{k}\right]$ is equivalent to $F L_{(k)} \rightarrow F K_{(k)} \perp\left[\mathrm{b}_{k}\right]$, and also to $F K_{(k)} \rightarrow$ $F L_{(k)} \perp\left[\mathrm{b}_{k}\right]$.

(ii) If $\mathfrak{f}_{k} \subset 4 \mathrm{a}_{k+1} \mathfrak{w}_{k+1}^{-1}$ and both $\mathrm{a}_{k+1}$ and $\mathrm{b}_{k+1}$ are norm generators for $L^{\mathfrak{s}_{k+1}}$ then $F L_{(k)} \rightarrow F K_{(k)} \perp\left[\mathrm{a}_{k+1}\right]$ is equivalent to $F L_{(k)} \rightarrow F K_{(k)} \perp\left[\mathrm{b}_{k+1}\right]$ and also to $F K_{(k)} \rightarrow F L_{(k)} \perp\left[\mathrm{b}_{k+1}\right]$.

Proof. (i) $\mathfrak{f}_{k} \subset 4 \mathrm{a}_{k} \mathfrak{w}_{k}^{-1}$ is equivalent to ord $\mathfrak{f}_{k}+\operatorname{ord} \mathrm{a}_{k}^{-1} \mathfrak{w}_{k}>2 e$. We have $\mathrm{a}_{k}^{-1} \mathfrak{w}_{k} \supseteq$ $2 \mathrm{a}_{k}^{-1} \mathfrak{s}_{k} \supseteq 2 \mathcal{O}$ so ord $\mathrm{a}_{k}^{-1} \mathfrak{w}_{k} \leq e<\operatorname{ord} \mathfrak{f}_{k}$. Since $\mathrm{a}_{k}, \mathrm{~b}_{k}$ are both norm generators for $L^{\mathfrak{s}_{k}}$ we have $d\left(\mathrm{a}_{k} \mathrm{~b}_{k}\right) \geq \operatorname{ord~a}_{k}^{-1} \mathfrak{w}_{k}$. Since also $d\left(\operatorname{det} L_{(k)} \operatorname{det} K_{(k)}\right) \geq$ $\operatorname{ord} \mathfrak{f}_{k}>\operatorname{ord} \mathrm{a}_{k}^{-1} \mathfrak{w}_{k}$ we also have $d\left(\mathrm{a}_{k} \mathrm{~b}_{k} \operatorname{det} L_{(k)} \operatorname{det} K_{(k)}\right) \geq \operatorname{ord} \mathrm{a}_{k}^{-1} \mathfrak{w}_{k}$. Since $d\left(\operatorname{det} L_{(k)} \operatorname{det} K_{(k)}\right)+d\left(\mathrm{a}_{k} \mathrm{~b}_{k}\right) \geq \operatorname{ord} \mathfrak{f}_{k}+\operatorname{ord} \mathrm{a}_{k}^{-1} \mathfrak{w}_{k}>2 e$ we get by Lemma 3.5(iii) that $F L_{(k)} \rightarrow F K_{(k)} \perp\left[\mathrm{a}_{k}\right]$ iff $F L_{(k)} \rightarrow F K_{(k)} \perp\left[\mathrm{b}_{k}\right]$. Similarly, since $d\left(\operatorname{det} L_{(k)} \operatorname{det} K_{(k)}\right)+d\left(\mathrm{a}_{k} \mathrm{~b}_{k} \operatorname{det} L_{(k)} \operatorname{det} K_{(k)}\right) \geq \operatorname{ord} \mathfrak{f}_{k}+\operatorname{ord} \mathrm{a}_{k}^{-1} \mathfrak{w}_{k}>2 e$, we have $F L_{(k)} \rightarrow F K_{(k)} \perp\left[\mathrm{a}_{k}\right]$ iff $F L_{(k)} \rightarrow F K_{(k)} \perp\left[\mathrm{b}_{k} \operatorname{det} L_{(k)} \operatorname{det} K_{(k)}\right]$ which, by Lemma 3.5 (ii), is equivalent to $F K_{(k)} \rightarrow F L_{(k)} \perp\left[\mathrm{b}_{k}\right]$.

(ii) The proof is the same as (i) but with $\mathrm{a}_{k}, \mathrm{~b}_{k}, \mathfrak{w}_{k}$ replaced by $\mathrm{a}_{k+1}, \mathrm{~b}_{k+1}, \mathfrak{w}_{k+1}$. 
Lemma 3.7. Suppose that $L, K$ satisfy the conditions 3.1(i) - (iii). If $1 \leq k \leq t-1$ then:

(i) If $\mathfrak{f}_{k} \subset 4 \mathrm{a}_{k} \mathfrak{w}_{k}^{-1}$ then $F L_{(k)} \rightarrow F K_{(k)} \perp\left[\mathrm{a}_{k}\right]$ iff $\left[b_{1}, \ldots, b_{i-1}\right] \rightarrow\left[a_{1}, \ldots, a_{i}\right]$, with $i=n_{k}$.

(ii) If $\mathfrak{f}_{k} \subset 4 \mathrm{a}_{k+1} \mathfrak{w}_{k+1}^{-1}$ then $F L_{(k)} \rightarrow F K_{(k)} \perp\left[\mathrm{a}_{k+1}\right]$ iff $\left[b_{1}, \ldots, b_{i-1}\right] \rightarrow$ $\left[a_{1}, \ldots, a_{i}\right]$, with $i=n_{k}+1$.

Proof. (i) We take $\mathrm{b}_{k}=-\pi^{2 u_{k}-2 r_{k}} b_{i}$ as a norm generator for $K^{\mathfrak{s}_{k}}$, so for $L^{\mathfrak{s}_{k}}$. (See Lemma 2.13(iii).) By Lemma 3.6(i) $F L_{(k)} \rightarrow F K_{(k)} \perp\left[\mathrm{a}_{k}\right]$ iff $F L_{(k)} \rightarrow F K_{(k)} \perp$ $\left[\mathrm{b}_{k}\right]$, i.e. iff $\left[a_{1}, \ldots, a_{i}\right] \rightarrow\left[b_{1}, \ldots, b_{i}\right] \perp\left[-b_{i}\right] \cong\left[b_{1}, \ldots, b_{i-1}\right] \perp$ H. By Lemma 3.5(i) this is equivalent to $\left[b_{1}, \ldots, b_{i-1}\right] \rightarrow\left[a_{1}, \ldots, a_{i}\right]$.

(ii) We take $\mathrm{b}_{k+1}=a_{i}$ as a norm generator for $L^{\mathfrak{s}_{k+1}}$. By Lemma 3.6(ii) $F L_{(k)} \rightarrow F K_{(k)} \perp\left[\mathrm{a}_{k+1}\right]$ iff $F K_{(k)} \rightarrow F L_{(k)} \perp\left[\mathrm{b}_{k+1}\right]$, i.e. iff $\left[b_{1}, \ldots, b_{i-1}\right] \rightarrow$ $\left[a_{1}, \ldots, a_{i-1}\right] \perp\left[a_{i}\right] \cong\left[a_{1}, \ldots, a_{i}\right]$.

Lemma 3.8. (i) If $i=n_{k}>n_{k-1}+1$ then $\alpha_{i-1}+\alpha_{i}>2 e$ iff $\mathfrak{f}_{k} \subset \mathrm{a}_{k} \mathfrak{w}_{k}^{-1}$.

(ii) If $i=n_{k}+1<n_{k+1}$ then $\alpha_{i-1}+\alpha_{i}>2 e$ iff $\mathfrak{f}_{k} \subset a_{k+1} \mathfrak{w}_{k+1}^{-1}$.

(iii) If $i=n_{k}=n_{k-1}+1$ then $\alpha_{i-1}+\alpha_{i}>2 e$ iff $\mathfrak{f}_{k} \subset \mathrm{a}_{k} \mathfrak{w}_{k}^{-1}$ or $\mathfrak{f}_{k-1} \subset \mathrm{a}_{k} \mathfrak{w}_{k}^{-1}$.

(In (iii) we ignore the condition $\mathfrak{f}_{k} \subset \mathrm{a}_{k} \mathfrak{w}_{k}^{-1}$ if $k=t$ and we ignore $\mathfrak{f}_{k-1} \subset \mathrm{a}_{k} \mathfrak{w}_{k}^{-1}$ if $k=1$.)

Proof. (i) Condition $\mathfrak{f}_{k} \subset 4 \mathrm{a}_{k} \mathfrak{w}_{k}^{-1}$ is equivalent to ord $\mathrm{a}_{k}^{-1} \mathfrak{w}_{k}+\operatorname{ord} \mathfrak{f}_{k}>2 e$. By Corollary 2.17(i) we have ord $\mathrm{a}_{k}^{-1} \mathfrak{w}_{k}=\alpha_{i-1}$. By Lemma 2.16(ii) we have either $\alpha_{i}=\operatorname{ord} \mathfrak{f}_{k}$ or $\alpha_{i}$, ord $\mathfrak{f}_{k}>2 e$. In the first case ord $\mathrm{a}_{k}^{-1} \mathfrak{w}_{k}+\operatorname{ord} \mathfrak{f}_{k}=\alpha_{i-1}+\alpha_{i}$ and in the second both ord $a_{k}^{-1} \mathfrak{w}_{k}+$ ord $\mathfrak{f}_{k}>2 e$ and $\alpha_{i-1}+\alpha_{i}>2 e$ hold. In both cases ord $a_{k}^{-1} \mathfrak{w}_{k}+\operatorname{ord} \mathfrak{f}_{k}>2 e$ iff $\alpha_{i-1}+\alpha_{i}>2 e$.

(ii) We have $\mathfrak{f}_{k} \subset 4 \mathrm{a}_{k+1} \mathfrak{w}_{k+1}^{-1}$ iff ord $\mathrm{a}_{k+1}^{-1} \mathfrak{w}_{k+1}+\operatorname{ord} \mathfrak{f}_{k}>2 e$. By Corollary 2.17(i) ord $\mathrm{a}_{k+1}^{-1} \mathfrak{w}_{k+1}=\alpha_{i}$ and by Lemma 2.16(ii) ord $\mathfrak{f}_{k}$ and $\alpha_{i-1}$ are either equal or they are both $>2 e$. Thus ord $a_{k+1}^{-1} \mathfrak{w}_{k+1}+$ ord $\mathfrak{f}_{k}>2 e$ iff $\alpha_{i-1}+\alpha_{i}>2 e$.

(iii) $\mathfrak{f}_{k-1} \subset 4 \mathrm{a}_{k} \mathfrak{w}_{k}^{-1}$ and $\mathfrak{f}_{k} \subset 4 \mathrm{a}_{k} \mathfrak{w}_{k}^{-1}$ are equivalent to ord $\mathfrak{f}_{k-1}+\operatorname{ord}_{k}^{-1} \mathfrak{w}_{k}>$ $2 e$, resp. ord $\mathfrak{f}_{k}+$ ord $\mathrm{a}_{k}^{-1} \mathfrak{w}_{k}>2 e$. By Corollary 2.17(ii) we have ord $\mathrm{a}_{k}^{-1} \mathfrak{w}_{k}=$ $\min \left\{\alpha_{i-1}, \alpha_{i}, e\right\} \geq 0$. By Lemma 2.16(ii) we have that ord $\mathfrak{f}_{k-1}=\alpha_{i-1}$ or ord $\mathfrak{f}_{k-1}, \alpha_{i-1}>2 e$ and ord $\mathfrak{f}_{k}=\alpha_{i}$ or ord $\mathfrak{f}_{k}, \alpha_{i}>2 e$. Therefore $\mathfrak{f}_{k-1} \subset 4 \mathrm{a}_{k} \mathfrak{w}_{k}^{-1}$ and $\mathfrak{f}_{k} \subset 4 \mathrm{a}_{k} \mathfrak{w}_{k}^{-1}$ are equivalent to $\alpha_{i-1}+\min \left\{\alpha_{i-1}, \alpha_{i}, e\right\}>2 e$, resp. $\alpha_{i}+$ $\min \left\{\alpha_{i-1}, \alpha_{i}, e\right\}>2 e$. Obviously either of them implies $\alpha_{i-1}+\alpha_{i}>2 e$. Conversely, suppose that $\alpha_{i-1}+\alpha_{i}>2 e$. If both $\alpha_{i-1}$ and $\alpha_{i}$ are $>e$ then we have both $\alpha_{i-1}+\min \left\{\alpha_{i-1}, \alpha_{i}, e\right\}>2 e$ and $\alpha_{i}+\min \left\{\alpha_{i-1}, \alpha_{i}, e\right\}>2 e$. Otherwise we have $\min \left\{\alpha_{i-1}, \alpha_{i}, e\right\}=\min \left\{\alpha_{i-1}, \alpha_{i}\right\}$ and so $\max \left\{\alpha_{i-1}, \alpha_{i}\right\}+\min \left\{\alpha_{i-1}, \alpha_{i}, e\right\}=$ $\max \left\{\alpha_{i-1}, \alpha_{i}\right\}+\min \left\{\alpha_{i-1}, \alpha_{i}\right\}=\alpha_{i-1}+\alpha_{i}>2 e$, which implies that either $\alpha_{i-1}+$ $\min \left\{\alpha_{i-1}, \alpha_{i}, e\right\}>2 e$ or $\alpha_{i}+\min \left\{\alpha_{i-1}, \alpha_{i}, e\right\}>2 e$.

Lemma 3.9. Assuming that 3.1(i)-(iii) hold, condition 3.1(iv) is equivalent to 93:28(ii) and (iii).

Proof. Take $1<i<n$. If $n_{k-1}+1<i<n_{k}$ for some $1 \leq k \leq t$ then $R_{i-1}=R_{i+1}$, by Lemma 2.13, so, by Lemma 3.4, $\alpha_{i-1}+\alpha_{i} \leq 2 e$, which makes 3.1(iv) vacuous at $i$. Therefore we can restrict ouselves to $i=n_{k}$ or $n_{k}+1$ for some $1 \leq k \leq t-1$. We have three cases:

1. $i=n_{k}$ and $\operatorname{dim} L_{k}>1$, i.e. $i=n_{k}>n_{k-1}+1$. By Lemma 3.8(i) $\mathfrak{f}_{k} \subset$ $4 \mathrm{a}_{k} \mathfrak{w}_{k}^{-1}$ is equivalent to $\alpha_{i-1}+\alpha_{i}>2 e$. On the other hand if $\mathfrak{f}_{k} \subset 4 \mathrm{a}_{k} \mathfrak{w}_{k}^{-1}$ then 
$F L_{(k)} \rightarrow F K_{(k)} \perp\left[\mathrm{a}_{k}\right]$ is equivalent to $\left[b_{1}, \ldots, b_{i-1}\right] \rightarrow\left[a_{1}, \ldots, a_{i}\right]$ by Lemma 3.7(i). Therefore 3.1(iv) at index $i$ is equivalent to 93:28(iii) at index $k$.

2. $i=n_{k}+1$ and $\operatorname{dim} L_{k+1}>1$, i.e. $i=n_{k}+1<n_{k+1}$. By Lemma 3.8(ii) $\mathfrak{f}_{k} \subset$ $4 \mathrm{a}_{k+1} \mathfrak{w}_{k+1}^{-1}$ is equivalent to $\alpha_{i-1}+\alpha_{i}>2 e$. On the other hand if $\mathfrak{f}_{k} \subset 4 \mathrm{a}_{k+1} \mathfrak{w}_{k+1}^{-1}$ then $F L_{(k)} \rightarrow F K_{(k)} \perp\left[\mathrm{a}_{k+1}\right]$ is equivalent to $\left[b_{1}, \ldots, b_{i-1}\right] \rightarrow\left[a_{1}, \ldots, a_{i}\right]$ by Lemma 3.7(ii). Therefore 3.1(iv) at index $i$ is equivalent to 93:28(ii) at index $k$.

3. $i=n_{k}=n_{k-1}+1$ for some $1 \leq k \leq t$. In this case $L_{k}$ is unary. We will prove that the condition 3.1(iv) at index $i$ is equivalent to 93:28(iii) at index $k$ and 93:28(ii) at index $k-1$. First note that if $k=t$ then 3.1(iv) is vacuous at $i=n_{t}=n$. On the other hand 93:28(iii) is vacuous at index $k=t$. Also if $\mathfrak{f}_{t-1} \subset 4 \mathrm{a}_{t} \mathfrak{w}_{t}^{-1}$ then, by Lemma 3.7(ii), $F L_{(t-1)} \rightarrow F K_{(t-1)} \perp\left[\mathrm{a}_{t}\right]$ is equivalent to $\left[b_{1}, \ldots, b_{n-1}\right] \rightarrow\left[a_{1}, \ldots, a_{n}\right]$ (we have $i=n_{t-1}+1=n_{t}=n$ ). But this follows from $\left[a_{1}, \ldots, a_{n}\right] \cong\left[b_{1}, \ldots, b_{n}\right]$. Thus 93:28(ii) is superfluous at index $k-1=t-1$. Next we note that if $k=1$ then 3.1 (iv) is vacuous at $i=n_{0}+1=1$. On the other hand 93:28(ii) is vacuous at index $k-1=0$. Also if $\mathfrak{f}_{1} \subset 4 \mathrm{a}_{1}^{-1} \mathfrak{w}_{1}$ then $F L_{(1)} \rightarrow F K_{(1)} \perp$ $\left[\mathrm{a}_{1}\right]$ is equivalent, by Lemma 3.7(i), to $0 \rightarrow\left[a_{1}\right]$ (we have $i=n_{1}=1$ ). Here 0 is not the scalar zero, but the zero lattice, of dimension 0 , so $0 \rightarrow\left[a_{1}\right]$ holds trivially. Thus 93:28(iii) is superfluous at $k=1$.

Now suppose that $1<k<t$. By Lemma 3.8(iii) we have $\alpha_{i-1}+\alpha_{i}>2 e$ iff $\mathfrak{f}_{k-1} \subset$ $4 \mathrm{a}_{k} \mathfrak{w}_{k}^{-1}$ or $\mathfrak{f}_{k} \subset 4 \mathrm{a}_{k} \mathfrak{w}_{k}^{-1}$. To complete the proof we note that if $\mathfrak{f}_{k-1} \subset 4 \mathrm{a}_{k} \mathfrak{w}_{k}^{-1}$ then $F L_{(k-1)} \rightarrow F K_{(k-1)} \perp\left[\mathrm{a}_{k}\right]$ is equivalent to $\left[b_{1}, \ldots, b_{i-1}\right] \rightarrow\left[a_{1}, \ldots, a_{i}\right]$ by Lemma 3.7(ii) (we have $i=n_{k-1}+1$ ) and if $\mathfrak{f}_{k} \subset 4 \mathrm{a}_{k} \mathfrak{w}_{k}^{-1}$ then $F L_{(k)} \rightarrow F K_{(k)} \perp\left[\mathrm{a}_{k}\right]$ is equivalent to $\left[b_{1}, \ldots, b_{i-1}\right] \rightarrow\left[a_{1}, \ldots, a_{i}\right]$ by Lemma 3.7(i) (we have $i=n_{k}$ ).

\section{THE 2-ADIC CASE}

In this section we will assume that $F$ is 2-adic, i.e. that $e=1$.

In [4, §93G] O'Meara gives a solution to the classification problem in the 2-adic case which only involves the Jordan invariants $t, \operatorname{dim} L_{k}, \mathfrak{s}_{k}$ and $\mathfrak{n}_{k}:=\mathfrak{n} L_{k}$. The invariants $\mathfrak{g}_{k}$ and $\mathfrak{w}_{k}$ are no longer necessary since they can be written as $\mathfrak{g}_{k}=\mathfrak{n}_{k}$ and $\mathfrak{w}_{k}=2 \mathfrak{s}_{k}$. A similar phenomenon occurs when we use good BONGs instead of Jordan decompositions. This time the invariants $\alpha_{i}$ are no longer necessary.

Lemma 4.1. If $e=1$ then $\alpha_{i}=1$ if $R_{i+1}-R_{i}=1$ and $\alpha_{i}=\left(R_{i+1}-R_{i}\right) / 2+1$ otherwise.

Proof. We have $R_{i+1}-R_{i} \geq-2 e=-2$ and if $R_{i+1}-R_{i}$ is negative then it is even. Thus $R_{i+1}-R_{i}$ is either -2 or it is $\geq 0$. If $R_{i+1}-R_{i}=-2 e=-2$ or $R_{i+1}-R_{i}=2 e-2=2-2 e=0$ or if $R_{i+1}-R_{i} \geq 2 e=2$ then $\alpha_{i}=$ $\left(R_{i+1}-R_{i}\right) / 2+e=\left(R_{i+1}-R_{i}\right) / 2+1$ by Corollary $2.9(\mathrm{i})$. If $R_{i+1}-R_{i}=1$, which is odd and $<2 e$, we have $\alpha_{i}=R_{i+1}-R_{i}=1$ by Lemma $2.7($ iii).

Since the $\alpha_{i}$ 's are uniquely defined by the $R_{i}$ 's, condition (ii) of the main theorem is superfluous since it follows from (i). Also, ord $a_{1} \cdots a_{i}=\operatorname{ord} b_{1} \cdots b_{i}$ so ord $a_{1} \cdots a_{i} b_{1} \cdots b_{i}$ is even. So if $R_{i+1}-R_{i} \leq 1$ we have $d\left(a_{1} \cdots a_{i} b_{1} \cdots b_{i}\right) \geq$ $1 \geq \alpha_{i}$. So condition (iii) is superfluous if $R_{i+1}-R_{i} \leq 1$. If $R_{i+1}-R_{i}=2$ then $\alpha_{2}=2$, while if $R_{i+1}-R_{i}>2$ then $\alpha_{i}>2$. Thus in these cases (iii) becomes $a_{1} \cdots a_{i} b_{1} \cdots b_{i} \in \dot{F}^{2} \cup \Delta \dot{F}^{2}$ if $R_{i+1}-R_{i}=2$ and $a_{1} \cdots a_{i} b_{1} \cdots b_{i} \in \dot{F}^{2}$ if $R_{i+1}-R_{i}>2$. Finally, it is easy to see that the condition $\alpha_{i-1}+\alpha_{i}>2$ from 
3.1(iv) is satisfied iff $R_{i-1}<R_{i+1}$ and the pair $\left(R_{i}-R_{i-1}, R_{i+1}-R_{i}\right)$ is different from $(0,1),(1,0),(1,1)$. So we have:

Theorem 4.2. Suppose that $F$ is 2 -adic, $L \cong \prec a_{1}, \ldots, a_{n} \succ$ and $K \cong \prec b_{1}, \ldots, b_{n} \succ$ relative to good BONGs, $R_{i}=R_{i}(L)=$ ord $a_{i}, S_{i}=R_{i}(K)=\operatorname{ord} b_{i}$ and $F L \cong F K$. Then $L \cong K$ if and only if the following conditions hold:

(i) $R_{i}=S_{i}$ for any $1 \leq i \leq n$.

(ii) For any $1 \leq i \leq n-1$ we have $a_{1} \cdots a_{i} b_{1} \cdots b_{i} \in \dot{F}^{2} \cup \Delta \dot{F}^{2}$ if $R_{i+1}-R_{i}=2$, and $a_{1} \cdots a_{i} b_{1} \cdots b_{i} \in \dot{F}^{2}$ if $R_{i+1}-R_{i}>2$.

(iii) $\left[b_{1}, \ldots, b_{i-1}\right] \rightarrow\left[a_{1}, \ldots, a_{i}\right]$ for any $1<i<n$ s.t. $R_{i-1}<R_{i+1}$ and $\left(R_{i}-\right.$ $\left.R_{i-1}, R_{i+1}-R_{i}\right) \neq(0,1),(1,0),(1,1)$.

\section{REMARKS}

\section{The binary case}

If $L \cong \prec \alpha, \beta \succ$ and $\eta \in \mathcal{O}^{\times}$then [1, 3.12] states that $L \cong \prec \eta \alpha, \eta \beta \succ$ iff $\eta \in g(a(L))=g\left(\frac{\beta}{\alpha}\right)$.

The function $g: \mathcal{A} \longrightarrow S g p\left(\mathcal{O}^{\times} / \mathcal{O}^{\times 2}\right)$ was introduced in [1, Definition 6]. Here $S g p H$ is the set of all subgroups of a group $H$. We recall the definition of $g$ 回

Definition. If $a=\pi^{R} \varepsilon \in \mathcal{A}$ and $d(-a)=d$ then:

I. If $R>2 e$ then $g(a)=\mathcal{O}^{\times 2}$.

II. If $R \leq 2 e$ then:

$$
g(a)= \begin{cases}\left(1+\mathfrak{p}^{R / 2+e}\right) \mathcal{O}^{\times 2} & \text { if } d>e-R / 2, \\ \left(1+\mathfrak{p}^{R+d}\right) \mathcal{O}^{\times 2} \cap \mathrm{N}(-a) & \text { if } d \leq e-R / 2 .\end{cases}
$$

The following lemma gives a more compact formula for $g(a)$.

Lemma 5.1. If $a \in \mathcal{A}$ and ord $a=R$ and $d(-a)=d$ then $g(a)=\left(1+\mathfrak{p}^{\alpha(a)}\right) \mathcal{O}^{\times^{2}} \cap$ $\mathrm{N}(-a)$, where $\alpha(a)=\min \{R / 2+e, R+d\}$.

Proof. By [1, 3.16] we have $g(a) \subseteq \mathrm{N}(-a)$. If $\eta \in \mathcal{O}^{\times}$then $\eta \in g(a)$ iff $\eta \in \mathrm{N}(-a)$ and (I) If $R>2 e$ then $\eta \in \mathcal{O}^{\times 2}$; (II) If $R \leq 2 e$ then $d(\eta) \geq R+d$ if $d \leq e-R / 2$, and $d(\eta) \geq R / 2+e$ if $d>e-R / 2$. (See [1, Definition 6].)

We have to prove that the conditions from (I) and (II) are equivalent to $d(\eta) \geq$ $\alpha(a)$. If $R>2 e$ then $R+d>2 e$ and $R / 2+e>2 e$ so $\alpha(a)>2 e$. Thus $d(\eta) \geq \alpha(a)$ is equivalent to $\eta \in \mathcal{O}^{\times 2}$. If $R \leq 2 e$ then $d \leq e-R / 2$ is equivalent to $R+d \leq R / 2+e$. Hence if $d \leq e-R / 2$ then $\alpha(a)=R+d$ and if $d>e-R / 2$ then $\alpha(a)=R / 2+e$.

If $n=2$ then from [1,3.12] we have $\prec a_{1}, a_{2} \succ \cong \prec \eta a_{1}, \eta a_{2} \succ$ iff $\eta \in g\left(a_{2} / a_{1}\right)$. By Lemma 5.1 this is equivalent to $\eta \in \mathrm{N}\left(-a_{1} a_{2}\right)$ and $d(\eta) \geq \alpha\left(a_{2} / a_{1}\right)$. The first condition is equivalent to the isometry of quadratic spaces $\left[a_{1}, a_{2}\right] \cong\left[\eta a_{1}, \eta a_{2}\right]$, while the second means $d(\eta) \geq \alpha\left(a_{2} / a_{1}\right)=\min \left\{\left(R_{2}-R_{1}\right) / 2+e, R_{2}-R_{1}+d\left(-a_{1} a_{2}\right)\right\}=$ $\alpha_{1}\left(\prec a_{1}, a_{2} \succ\right.$ ), which is consistent with condition (iii) of the main theorem.

Remark 5.2. Since $\alpha\left(a_{2} / a_{1}\right)=\alpha_{1}\left(\prec a_{1}, a_{2} \succ\right)$ we have by Lemma $5.1 g\left(a_{2} / a_{1}\right)=$ $\left(1+\mathfrak{p}^{\alpha_{1}\left(\prec a_{1}, a_{2} \succ\right)}\right) \mathcal{O}^{\times 2} \cap \mathrm{N}\left(-a_{1} a_{2}\right)$. Equivalently, $g(a(L))=\left(1+\mathfrak{p}^{\alpha_{1}(L)}\right) \mathcal{O}^{\times 2} \cap$ $\mathrm{N}(-\operatorname{det} F L)$.

\footnotetext{
${ }^{1}$ In [1 Definition 6] there are some mistakes which we corrected here.
} 


\section{The formula for $\alpha_{i}$}

We will now show the heuristical method by which the invariants $\alpha_{i}$ were found. We want to know, given that $L \cong \prec a_{1}, \ldots, a_{n} \succ$ relative to a good BONG and $1 \leq i \leq n-1$, how much the product $a_{1} \cdots a_{i}$ can be altered by a change of good BONGs. That is, if $L \cong \prec b_{1}, \ldots, b_{n} \succ$ relative to another good BONG we want to know how big the quadratic defect of $\left(b_{1} \cdots b_{i}\right) /\left(a_{1} \cdots a_{i}\right)$ can be. So we are looking for a lower bound $\alpha_{i}=\alpha_{i}(L)$ for $d\left(a_{1} \cdots a_{i} b_{1} \cdots b_{i}\right)$.

For any $\eta \in g\left(a_{i+1} / a_{i}\right)$ we have $\prec a_{i}, a_{i+1} \succ \cong \prec \eta a_{i}, \eta a_{i+1} \succ$ so, by [1, Lemma 4.9(ii)], $L \cong \prec a_{1}, \ldots, a_{i-1}, \eta a_{i}, \eta a_{i+1}, a_{i+2}, \ldots, a_{n} \succ$. By this change of BONGs, $a_{1} \cdots a_{i}$ was changed by the factor $\eta$. We have $\eta \in g\left(a_{i+1} / a_{i}\right)$ which, by Lemma 5.1 , implies $d(\eta) \geq \alpha\left(a_{i+1} / a_{i}\right)=\min \left\{\left(R_{i+1}-R_{i}\right) / 2+e, R_{i+1}-R_{i}+d\left(-a_{i} a_{i+1}\right)\right\}$. (See Lemma 5.1.) This lower bound can be further decreased if we decrease $d\left(-a_{i} a_{i+1}\right)$. This can be done by changing the good BONGs of $\prec a_{1}, \ldots, a_{i} \succ$ and $\prec a_{i+1}, \ldots, a_{n} \succ$. If $\prec a_{1}, \ldots, a_{i} \succ \cong \prec a_{1}^{\prime}, \ldots, a_{i}^{\prime} \succ$ and $\prec a_{i+1}, \ldots, a_{n} \succ \cong$ $\prec a_{i+1}^{\prime}, \ldots, a_{n}^{\prime} \succ$ then $d\left(-a_{i} a_{i+1}\right)$ is replaced by $d\left(-a_{i}^{\prime} a_{i+1}^{\prime}\right)$. But $d\left(a_{i+1} a_{i+1}^{\prime}\right) \geq$ $\alpha_{1}\left(\prec a_{i+1}, \ldots, a_{n} \succ\right)$. Also, by reason of determinant, $a_{1} \cdots a_{i} a_{1}^{\prime} \cdots a_{i}^{\prime} \in \dot{F}^{2}$ so $d\left(a_{i} a_{i}^{\prime}\right)=d\left(a_{1} \cdots a_{i-1} a_{1}^{\prime} \cdots a_{i-1}^{\prime}\right) \geq \alpha_{i-1}\left(\prec a_{1}, \ldots, a_{i} \succ\right)$. It follows that $d\left(-a_{i}^{\prime} a_{i+1}^{\prime}\right) \geq \min \left\{d\left(-a_{i} a_{i+1}\right), \alpha_{i-1}\left(\prec a_{1}, \ldots, a_{i} \succ\right), \alpha_{1}\left(\prec a_{i+1}, \ldots, a_{n} \succ\right)\right\}$. Hence the new lower bound for $\eta$ is $\min \left\{\left(R_{i+1}-R_{i}\right) / 2+e, R_{i+1}-R_{i}+d\left(-a_{i} a_{i+1}\right), R_{i+1}-\right.$ $\left.R_{i}+\alpha_{i-1}\left(\prec a_{1}, \ldots, a_{i} \succ\right), R_{i+1}-R_{i}+\alpha_{1}\left(\prec a_{i+1}, \ldots, a_{n} \succ\right)\right\}$. This leads to the recursive formula $\alpha_{i}=\min \left\{\left(R_{i+1}-R_{i}\right) / 2+e, R_{i+1}-R_{i}+d\left(-a_{i} a_{i+1}\right), R_{i+1}-R_{i}+\right.$ $\left.\alpha_{i-1}\left(\prec a_{1}, \ldots, a_{i} \succ\right), R_{i+1}-R_{i}+\alpha_{1}\left(\prec a_{i+1}, \ldots, a_{n} \succ\right)\right\}$ from Corollary 2.5 (ii).

In the case $i=1$ and $n \geq 3$ the formula becomes $\alpha_{1}=\min \left\{\left(R_{2}-R_{1}\right) / 2+e, R_{2}-\right.$ $\left.R_{1}+d\left(-a_{1} a_{2}\right), R_{2}-R_{1}+\alpha_{1}\left(\prec a_{2}, \ldots, a_{n} \succ\right)\right\}$. In the case $i=n-1$ and $n \geq 3$ we have $\alpha_{n-1}=\min \left\{\left(R_{n}-R_{n-1}\right) / 2+e, R_{n}-R_{n-1}+d\left(-a_{n-1} a_{n}\right), R_{n}-R_{n-1}+\right.$ $\left.\alpha_{n-2}\left(\prec a_{1}, \ldots, a_{n-1} \succ\right)\right\}$. Finally if $i=1$ and $n=2$ then $\alpha_{1}=\min \left\{\left(R_{2}-R_{1}\right) / 2+\right.$ $\left.e, R_{2}-R_{1}+d\left(-a_{1} a_{2}\right)\right\}$. Starting with the case $n=2$ it is easy to prove by induction that $\alpha_{1}=\min \left(\left\{\left(R_{2}-R_{1}\right) / 2+e\right\} \cup\left\{R_{j+1}-R_{1}+d\left(-a_{j} a_{j+1}\right) \mid 1 \leq j<n\right\}\right)$ and $\alpha_{n-1}=\min \left(\left\{\left(R_{n}-R_{n-1}\right) / 2+e\right\} \cup\left\{R_{n}-R_{j}+d\left(-a_{j} a_{j+1}\right) \mid 1 \leq j<n\right\}\right)$. By plugging $\alpha_{i-1}\left(\prec a_{1}, \ldots, a_{i} \succ\right)=\min \left(\left\{\left(R_{i}-R_{i-1}\right) / 2+e\right\} \cup\left\{R_{i}-R_{j}+d\left(-a_{j} a_{j+1}\right) \mid\right.\right.$ $1 \leq j<i\})$ and $\alpha_{1}\left(\prec a_{i+1}, \ldots, a_{n} \succ\right)=\min \left(\left\{\left(R_{i+2}-R_{i+1}\right) / 2+e\right\} \cup\left\{R_{j+1}-\right.\right.$ $\left.\left.R_{i+1}+d\left(-a_{j} a_{j+1}\right) \mid i+1 \leq j<n\right\}\right)$ in the recursive formula for $\alpha_{i}$ we get the formula from Definition 1. (The extra terms $R_{i+1}-R_{i}+\left(R_{i}-R_{i-1}\right) / 2+e$ and $R_{i+1}-R_{i}+\left(R_{i+2}-R_{i+1}\right) / 2+e$ that appear are $\geq\left(R_{i+1}-R_{i}\right) / 2+e$ so they can be removed.)

Of course this is only a guess and does not constitute a proof. In fact the relation $d\left(a_{1} \cdots a_{i} b_{1} \cdots b_{i}\right) \geq \alpha_{i}$ is only proved this way in the particular case when $b_{1}, \ldots, b_{n}$ are obtained from $a_{1}, \ldots, a_{n}$ through a succession of "binary transformations" of the type $a_{1}, \ldots, a_{n} \longrightarrow a_{1}, \ldots, \eta a_{j}, \eta a_{j+1}, \ldots, a_{n}$ with $1 \leq j \leq n-1$ and $\eta \in$ $g\left(a_{j+1} / a_{j}\right)$. It is not hard to prove that conditions (i)-(iv) of the main theorem are necessary if $b_{1}, \ldots, b_{n}$ are obtained this way. However, for the proof of the necessity in the general case and for the proof of sufficiency the use of O'Meara's theorem is necessary.

3. In the view of the previous remark there is the natural question that asks whether, given that $L \cong \prec a_{1}, \ldots, a_{n} \succ \cong \prec b_{1}, \ldots, b_{n} \succ$ relative to good BONGs, there is always a succession of binary transformations as defined above from $a_{1}, \ldots$, $a_{n}$ to $b_{1}, \ldots, b_{n}$. The answer to this question is YES but only if we make the 
assumption that $F / \mathbb{Q}_{2}$ is not totally ramified, i.e. that the residual field $\mathcal{O} / \mathfrak{p}$ has more than 2 elements.

If $|\mathcal{O} / \mathfrak{p}|=2$ we have the following counterexample. Let $0<d<2 e$ be odd and let $R=2 e-2 d$ and $\varepsilon, \eta \in \mathcal{O}^{\times}$with $d(\varepsilon)=d$ and $d(\eta)=2 e-d$. It can be proved that $\prec 1,-\pi^{R} \varepsilon, \varepsilon \eta,-\pi^{R} \eta \succ \cong \prec \eta,-\pi^{R} \varepsilon \eta, \varepsilon,-\pi^{R} \succ$ but one cannot go from $1,-\pi^{R} \varepsilon, \varepsilon \eta,-\pi^{R} \eta$ to $\eta,-\pi^{R} \varepsilon \eta, \varepsilon,-\pi^{R}$ through binary transformations.

E.g., if $F=\mathbb{Q}_{2}$ and we take $d=1$, so $R=0$, and $\varepsilon=\eta=-1$ then $\prec 1,1,1,1 \succ \cong \prec 7,7,7,7 \succ$. However from $1,1,1,1$ we can go through binary transformations only to $a_{1}, a_{2}, a_{3}, a_{4}$, where an even number of $a_{i}$ 's belong to $\mathcal{O}^{\times 2}$ and the rest to $5 \mathcal{O}^{\times 2}$. This happens because $g(1)=g(5)=\mathcal{O}^{\times 2} \cup 5 \mathcal{O}^{\times 2}$ so the only binary relations involving 1 and 5 are $\prec 1,1 \succ \cong \prec 5,5 \succ$ and $\prec 1,5 \succ \cong \prec 5,1 \succ$. Similarly from $7,7,7,7$ we can only go to $a_{1}, a_{2}, a_{3}, a_{4}$, where an even number of $a_{i}$ 's belong to $7 \mathcal{O}^{\times 2}$ and the rest to $3 \mathcal{O}^{\times 2}$.

\section{REFERENCES}

[1] C. N. Beli, Integral spinor norms over dyadic local fields, J. Number Theory 102 (2003) 125-182. MR.1994477 (2004i:11030)

[2] C.N Beli, Representations of integral quadratic forms over dyadic local fields, Electronic Research Announcements of the American Mathematical Society 12, 100-112, electronic only (2006). MR 2237274 (2007m:11054)

[3] J. S. Hsia, Spinor norms of local integral rotations I, Pacific J. Math., Vol. 57 (1975), 199 206. MR0374029(51:10229)

[4] O. T. O'Meara, Introduction to Quadratic Forms, Springer-Verlag, Berlin (1963). MR0152507 $(27: 2485)$

Institute of Mathematics of the Romanian Academy, P.O. Box 1-764, RO-70700 Bucharest, Romania

E-mail address: raspopitu1@yahoo.com

E-mail address: Constantin.Beli@imar.ro 